\title{
Identification and Characterization of Fungal Pathogens Causing Fruit Rot of Deciduous Holly
}

Shan Lin, Department of Plant Pathology, The Ohio State University, Columbus, OH 43210; Nancy J. Taylor, C. Wayne Ellett Plant and Pest Diagnostic Clinic, The Ohio State University, Reynoldsburg, OH 43068; and Francesca Peduto Hand, ${ }^{\dagger}$ Department of Plant Pathology, The Ohio State University, Columbus, OH 43210

\begin{abstract}
Cut branches of deciduous holly (Ilex spp. L.) harboring colorful berries are traditionally used as ornaments in holiday decorations. Since 2012, a fruit rot of unspecified cause has resulted in significant yield reduction and economic losses across Midwestern and Eastern U.S. nurseries. In this study, symptomatic fruit samples collected from nine different locations over five years were analyzed, and several fungal species were isolated. A combination of morphological characterization, multilocus phylogenetic analyses, and pathogenicity assays revealed that Alternaria alternata and Diaporthe ilicicola sp. nov. were the primary pathogens associated with symptomatic fruit. Other fungi including A. arborescens, Colletotrichum fioriniae, C. nymphaeae, Epicoccum nigrum, and species

in the $D$. eres species complex appeared to be minor pathogens in this disease complex. In detached fruit pathogenicity assays testing the role of wounding and inoculum concentration on disease development, disease incidence and severity increased when fruit was wounded and inoculated with a higher inoculum concentration. These findings indicate that management strategies that can protect fruit from injury or reduce inoculum may lower disease levels in the field. This research established the basis for further studies on this emerging disease and the design of research-based management strategies. To our knowledge, it also represents the first report of species of Alternaria, Colletotrichum, Diaporthe, and Epicoccum causing fruit rot of deciduous holly.
\end{abstract}

Members of the genus Ilex are deciduous or evergreen ornamental shrubs or trees, ranging in height from 1 to over $30 \mathrm{~m}$ (Galle 1997). The deciduous species are popularly used in the cut flower industry for winter holiday decorations because of the beautiful display of colorful fruit they present, varying from red to orange or yellow depending on the hybrid and cultivar, that can persist on the plants throughout the winter (Fig. 1A). The overall sale value of Ilex spp. in the cut flower industry is estimated at $\$ 571,279$ nationally (NASS 2014). Sales of the cut woody stems allows revenue in late fall and early winter when there is little activity and little other income for this segment of the nursery industry, also contributing to extended employment for seasonal employees.

The three most common deciduous holly species grown in North America are Ilex decidua (possumhaw holly), I. serrata (Japanese winterberry), and I. verticillata (common winterberry), along with their many cultivars and hybrids. Plants are functionally polygamodioecious (mostly dioecious, but with either a few flowers of the opposite sex or a few bisexual flowers on the same plant [Fernald 1950]), requiring both male and female plants with similar flowering periods in the same field for pollination. The fruit of Ilex is composed of chartaceous exocarp, juicy mesocarp, and separated coriaceous endocarp. From a botanical perspective, it is neither a berry nor a drupe, and there is a lack of specific terminology for its designation. Although the term "bacco-drupe" was proposed by Hu in 1950 (Galle

${ }^{\dagger}$ Corresponding author: F. Peduto Hand; E-mail: hand.81@osu.edu

Funding: This publication was financed in part or totally through a grant from the Ohio Department of Agriculture Specialty Crop Promotion Program, the State of Ohio, and the United States Department of Agriculture under the provisions of the Specialty Crop Block Grant. This project was supported by the Specialty Crop Block Grant Program at the U.S. Department of Agriculture through grants AGR-SCG-14-08 and AGR-SCG-16-09. This study was also funded by the USDA-NIFA Hatch project \#1004939, the T. J. Kavanagh Foundation, and The Ohio State University Department of Plant Pathology.

This paper's contents are solely the responsibility of the authors and do not necessarily represent the official views of the USDA.

Accepted for publication 11 May 2018.

() 2018 The American Phytopathological Society
1997), there has been a lack of agreement on the use of this term. Therefore, the general term "fruit" will be used throughout this manuscript to refer to the fruit of Ilex.

Since 2012, a fruit rot of unspecified cause has been challenging holly growers across the Midwestern and Eastern United States. Affected plants defoliate earlier in the season (September rather than October) and carry undersized fruit that fails to turn color, loses its normal gloss, shrivels, and eventually becomes necrotic (Fig. 1B and C). Some popular cultivars, including Bonfire, Sparkleberry, and Winter Red, are particularly affected. As a consequence, nursery growers are reporting decreased crop yield and, in some cases, complete crop loss. While an official value that quantifies these losses is not available in the literature, in the winter of 2014, several Ohio growers who participated in this project lost their entire crop to this fruit rot (Peduto Hand, personal observation).

In the winter of 2013, at the time of branch harvest, a few symptomatic fruit samples from three Ohio nurseries were sent to the C. Wayne Ellett Plant and Pest Diagnostic Clinic at The Ohio State University for diagnosis. The involvement of bacteria and oomycetes was ruled out through the diagnostic process but several different fungi were isolated from the symptomatic tissues, belonging to the genera Alternaria Nees, Colletotrichum Corda, Diaporthe Nitschke., and undetermined genera in the Botryosphaeriaceae Theiss. \& P. Syd. Several species within these fungi are well known and economically important fruit rot pathogens that have been extensively studied on many fruit crops, including apple (Biggs 2004; Gao et al. 2013; Munir et al. 2016), blueberry (Milholland and Daykin 1983; Zhu and Xiao 2015), cranberry (Olatinwo et al. 2003), and strawberry (Curry et al. 2002; Howard et al. 1992). To date, there is no official record of the occurrence of the above fungal genera as pathogens causing fruit rot on deciduous holly.

Understanding this disease problem, including elucidating the components of the disease triangle (pathogen-host-environment), is a critical step toward providing nursery growers with researchbased recommendations for effective management. Thus, the initial objectives of our investigation were to (i) confirm identification of the fungal pathogen(s) associated with the disease from samples collected over multiple years and across multiple locations by conducting morphological and molecular studies, and (ii) determine factors that might be contributing to fruit infection. Future research will build upon these findings and will focus on elucidating the sources of pathogen inoculum in the field, the timing of host susceptibility 
to infection, as well as the role of several environmental factors in disease development. This information will be critical to identify potential management measures, including appropriate timing for horticultural practices and fungicide treatments.

\section{Materials and Methods}

Sample collection and fungal isolation. Between 2013 and 2017, 623 symptomatic fruit of deciduous holly comprising cultivars Bonfire, Oosterwijk, Sparkleberry, and Winter Red were received or collected from commercial nurseries, landscapes, and arboreta across the Midwestern and Eastern U.S., including Ohio $(n=$ 593), Massachusetts $(n=10)$, North Carolina $(n=15)$, and Pennsylvania $(n=5)$. Samples received from out of state were processed immediately upon receipt, while those collected from field sites in Ohio were transported to the laboratory within a cooler and then kept at $4{ }^{\circ} \mathrm{C}$ for a maximum of $24 \mathrm{~h}$ before being processed. Samples were surface disinfected with $2 \%$ Clorox household bleach (The Clorox Company) for $2 \mathrm{~min}$, then rinsed in sterile water three times. Following disinfection, samples were air dried on sterile paper towels in a laminar flow hood. Two pieces of symptomatic tissue comprising the exocarp and mesocarp were excised from the edge of each lesion with a sterile scalpel and transferred to potato dextrose agar (PDA; Difco Laboratories, Sparks, MD) amended with $0.01 \%$ tetracycline hydrochloride and $0.015 \%$ streptomycin sulfate (Fisher Scientific, Fair Lawn, NJ). All plates were incubated at $25^{\circ} \mathrm{C}$ under constant light for up to 10 days. Plates were checked daily and any observed fungal growth was subcultured onto PDA. Pure cultures of each isolate were obtained by transferring hyphal tips from the margin of the fungal colonies onto new PDA plates. All isolates were then grown on sterile filter paper and stored at $-20^{\circ} \mathrm{C}$ until further use.

Morphological characterization. Isolates were first identified to genus based on comparison of morphological characteristics of the spores and spore-producing structures with descriptions available in the literature (Barnett and Hunter 1998). Two to 14 isolates of each of the most represented fungal genera (i.e., $>4 \%$ isolation frequency) were then selected to include all locations $(n=9)$ and years $(n=5)$ of sampling and subject to further characterization (Table 1). Pure cultures of all isolates except Alternaria spp. were transferred to fresh PDA and incubated at $23^{\circ} \mathrm{C}$ with a 12 -h photoperiod for up to 4 weeks to induce sporulation. Isolates tentatively identified as Alternaria spp. were grown on V8 juice agar as described by Simmons (2007).
The length and width of 30 randomly selected conidia from each isolate included in the study (Table 1) were measured using a Leica DM750 compound microscope (Leica Microsystems CMS GmbH, Wetzlar, Germany) and the Leica Application Suite EZ software (version 3.0.0). Mean and standard error of the conidial measurements were calculated for each isolate. Conidial color, shape, and presence or absence of septation were also recorded. Additionally, fungal colony diameter on each of three plates per fungal isolate was measured 3, 5, and 7 days post-inoculation (DPI) to calculate mycelial growth rates $\left(\mathrm{mm} \mathrm{day}^{-1}\right)$. All data were analyzed independently using PROC GLM in SAS (version 9.4; SAS Institute Inc., Cary NC). Tukey's honest significant difference $(\alpha=0.05)$ was used for mean comparisons among isolates.

DNA extraction, amplification, and sequencing. Total genomic DNA was extracted from pure cultures of isolates grown on PDA at $25^{\circ} \mathrm{C}$ for 7 days using the DNeasy Plant Mini Kit (Qiagen Inc., Hilden, Germany) according to manufacturer's instructions. All PCR amplifications were carried out in a final volume of $20 \mu \mathrm{l}$ using the Bio-Rad T100 Thermal Cycler (Bio-Rad Laboratories, Inc., Hercules, CA). All amplified loci, primers, and PCR conditions are summarized in Table 2. Amplified PCR products were resolved by electrophoresis at $100 \mathrm{~V} / \mathrm{cm}$ in $1.5 \%$ agarose gels in $1 \times$ Tris-borate-EDTA buffer stained with $0.005 \%$ GelRed (Biotium Inc., Fremont, CA) and visualized under a UV transilluminator. The size of the fragments was estimated using a 1-kb DNA ladder (New England BioLabs Inc., Ipswich, MA). PCR products were purified using ExoSAP-IT (Affymetrix, Inc., Santa Clara, CA) according to manufacturer's instructions and both strands were sequenced at the Genomics Shared Resource at the Ohio State University Comprehensive Cancer Center using an ABI Prism 3730 DNA analyzer (Applied Biosystems, Foster City, CA). Sequences were manually edited using the software Chromas (v 2.6.4, Technelysium Pty Ltd, Australia) and assembled using MUSCLE in MEGA7 (Kumar et al. 2016). Consensus sequences of each locus were exported and compared with those available in GenBank using the Basic Local Alignment Search Tool (BLAST; National Center for Biotechnology Information, Bethesda, MD).

Phylogenetic analyses. The same isolates selected for morphological characterization were subject to phylogenetic analyses (Table 1). Sequences of reference strains recently published in the literature were retrieved from GenBank and included in the analysis for

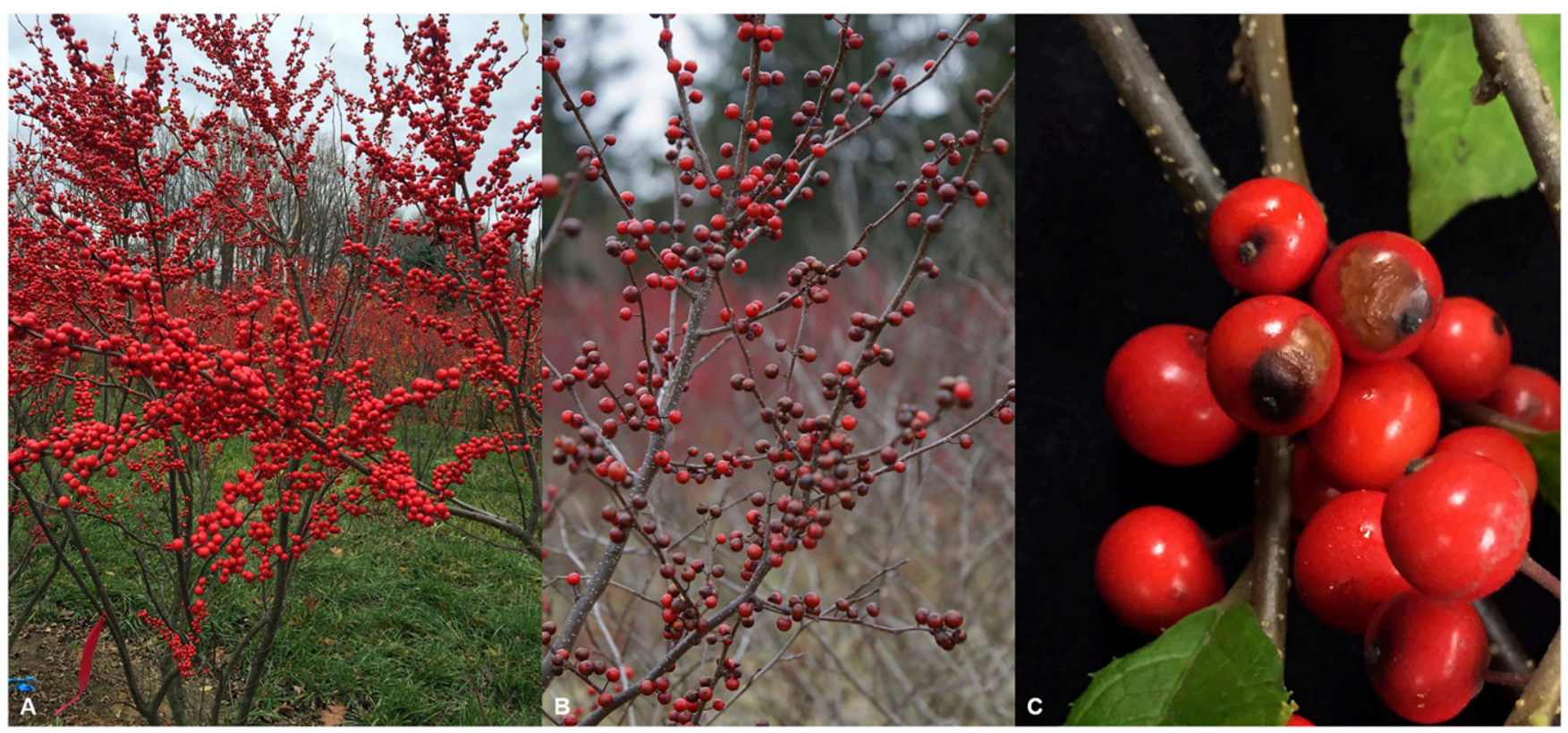

Fig. 1. Fruit rot symptoms observed on deciduous holly cultivar Sparkleberry in an Ohio nursery. A, healthy plants; B, overview of symptomatic berries on an entire plant; C, closeup of fruit exhibiting rot symptoms on twigs. 
comparison (Table 3; Andrew et al. 2009; Damm et al. 2012; Dissanayake et al. 2017; Du et al. 2016; Gomes et al. 2013; Huang et al. 2013; Huang et al. 2015; Machingambi et al. 2015; Mostert et al. 2001; Peever et al. 2004; Rotondo et al. 2012; Tan et al. 2013; Thompson et al. 2015; Udayanga et al. 2012, 2014a, b, 2015; Valenzuela-Lopez et al. 2018; Woudenberg et al. 2015; Yang et al. 2017). Concatenated sequences of different loci for each fungal genus (endoPG, OPA1, and OPA10 for Alternaria; TUB and GADPH for Colletotrichum; ITS, HIS, and TUB for Diaporthe) were aligned using MUSCLE in MEGA7 (Kumar et al. 2016). The nucleotide substitution model for each dataset was determined using FindModel (https://www.hiv.lanl.gov/content/sequence/findmodel/findmodel. html) based on the Akaike Information Criterion (AIC). Each concatenated dataset was subjected to maximum likelihood (ML) analysis in MEGA7 and to Bayesian inference (BI) analysis using MrBayes as implemented in siMBa (Mishra and Thines 2014; Ronquist and Huelsenbeck 2003). The heuristic method in ML analysis used subtree-pruning-regrafting with SPR level 5, and branch strength was evaluated using 1,000 bootstrap replications. All gaps and missing data were deleted. For BI, a Metropolis Coupled Markov Chain Monte Carlo (MCMCMC) analysis was carried out with two independent runs with four chains each for one million generations. The analysis was stopped when the average standard deviation of split frequencies fell below 0.01 . Trees were sampled every 500 generations and the first $25 \%$ of samples were discarded as "burn-in." Trees were visualized in FigTree v. 1.4.3 (Rambaut 2016).

Representative sequences for each locus and pathogen species included in this study were deposited into GenBank (Table 1). All isolate cultures are maintained in the fungal collection of the Department of Plant Pathology at The Ohio State University. The novel taxon described in this study was also deposited in the Westerdijk Fungal Biodiversity Institute Collection (CBS-KNAW, Utrecht, The Netherlands) and registered in MycoBank (www.mycobank.org).

Pathogenicity tests. Three sets of pathogenicity experiments, each conducted twice, were carried out to further characterize the isolates included in Table 1. In the first experiment (A), healthy fruit were collected from potted plants of cultivar Sparkleberry and surface-disinfested as previously described. Ten individual fruit were

Table 1. Fungal isolates recovered from symptomatic holly fruit used in this study for morphological characterization, phylogenetic analyses, and pathogenicity tests

\begin{tabular}{|c|c|c|c|c|c|c|c|}
\hline \multirow[b]{2}{*}{ Species } & \multirow[b]{2}{*}{ Origin $^{x}$} & \multirow[b]{2}{*}{ Year } & \multirow[b]{2}{*}{ Isolate $^{y}$} & \multirow[b]{2}{*}{ Host, cultivar } & \multicolumn{3}{|c|}{ GenBank accession no. ${ }^{\mathrm{z}}$} \\
\hline & & & & & EPG & OPA1 & OPA10 \\
\hline Alternaria alternata & MA & 2015 & FPH2015390 & Ilex verticillata, Oosterwijk & MH003571 & MH003585 & MH003599 \\
\hline A. alternata & MA & 2015 & FPH2015396 & I. verticillata, Oosterwijk & MH003573 & МH003587 & MH003601 \\
\hline A. alternata & $\mathrm{OH}$ & 2015 & FPH2015338 & I. verticillata $\times$ I. serrata, Sparkleberry & MH003570 & МH003584 & MH003598 \\
\hline A. alternata & $\mathrm{OH}$ & 2015 & FPH2015468 & I. verticillata $\times$ I. serrata, Bonfire & MH003574 & МH003588 & MH003602 \\
\hline A. alternata & $\mathrm{OH}$ & 2015 & FPH2015470 & I. verticillata $\times I$. serrata, Sparkleberry & MH003575 & MH003589 & MH003603 \\
\hline A. alternata & $\mathrm{OH}$ & 2015 & FPH2015507* & I. verticillata $\times$ I. serrata, Sparkleberry & MH003576 & МH003590 & MH003604 \\
\hline A. alternata & $\mathrm{OH}$ & 2015 & FPH2015567 & I. verticillata $\times$ I. serrata, Sparkleberry & MH003577 & MH003591 & MH003605 \\
\hline A. alternata & $\mathrm{OH}$ & 2015 & FPH2015589 & I. verticillata $\times$ I. serrata, Bonfire & MH003578 & MH003592 & MH003606 \\
\hline A. alternata & $\mathrm{OH}$ & 2015 & FPH2015591 & I. verticillata $\times$ I. serrata, Bonfire & МH003579 & МH003593 & MH003607 \\
\hline A. alternata & $\mathrm{OH}$ & 2015 & FPH2015592 & I. verticillata $\times$ I. serrata, Bonfire & MH003580 & MH003594 & MH003608 \\
\hline A. alternata & $\mathrm{OH}$ & 2016 & FPH2015597 & I. verticillata $\times$ I. serrata, Sparkleberry & MH003581 & MH003595 & MH003609 \\
\hline A. alternata & $\mathrm{OH}$ & 2016 & FPH2016709 & I. verticillata $\times I$. serrata, Bonfire & MH003582 & МH003596 & MH003610 \\
\hline A. alternata & $\mathrm{OH}$ & 2016 & FPH2016711 & I. verticillata $\times I$. serrata, Bonfire & МH003583 & MH003597 & MH003611 \\
\hline A. arborescens & MA & 2015 & FPH2015395 & I. verticillata, Oosterwijk & $\begin{array}{c}\text { MH003572 } \\
\text { GAPDH }\end{array}$ & $\begin{array}{c}\text { MH003586 } \\
\text { TUB }\end{array}$ & MH003600 \\
\hline Colletotrichum fioriniae & MA & 2015 & FPH2015392 & I. verticillata, Oosterwijk & MH003615 & МH003623 & MH003615 \\
\hline C. fioriniae & $\mathrm{OH}$ & 2013 & FPH201317 & I. verticillata $\times I$. serrata, Sparkleberry & MH003613 & МH003621 & MH003613 \\
\hline C. fioriniae & $\mathrm{OH}$ & 2013 & FPH201318 & I. verticillata $\times$ I. serrata, Sparkleberry & МH003614 & МH003622 & MH003614 \\
\hline C. fioriniae & $\mathrm{OH}$ & 2015 & FPH2015466* & I. verticillata $\times$ I. serrata, Bonfire & MH003616 & МH003624 & MH003616 \\
\hline C. fioriniae & $\mathrm{OH}$ & 2015 & FPH2015564 & I. verticillata $\times I$. serrata, Bonfire & MH003617 & МH003625 & MH003617 \\
\hline C. fioriniae & $\mathrm{OH}$ & 2016 & FPH2016720 & I. verticillata $\times$ I. serrata, Sparkleberry & MH003619 & МH003627 & MH003619 \\
\hline C. fioriniae & $\mathrm{OH}$ & 2013 & FPH201314 & I. verticillata $\times$ I. serrata, Sparkleberry & MH003612 & MH003620 & MH003612 \\
\hline C. nymphaeae & $\mathrm{OH}$ & 2015 & FPH2015590 & I. verticillata $\times$ I. serrata, Bonfire & $\begin{array}{c}\text { MH003618 } \\
\text { HIS }\end{array}$ & $\begin{array}{c}\text { MH003626 } \\
\text { ITS }\end{array}$ & $\begin{array}{c}\text { MH003618 } \\
\text { TUB }\end{array}$ \\
\hline Diaporthe eres & MA & 2015 & FPH2015394 & I. verticillata, Oosterwijk & MH003629 & MH003632 & MH003635 \\
\hline D. eres & $\mathrm{OH}$ & 2013 & FPH201322 & I. verticillata $\times$ I. serrata, Bonfire & MH003628 & MH003631 & MH003634 \\
\hline D. eres & $\mathrm{PA}$ & 2017 & FPH2017229 & I. verticillata $\times$ I. serrata, Bonfire & МH003630 & МH003633 & MH003636 \\
\hline D. ilicicola sp. nov. & $\mathrm{OH}$ & 2015 & FPH2015472 & I. verticillata $\times$ I. serrata, Sparkleberry & MH171082 & MH171062 & MH171072 \\
\hline D. ilicicola sp. nov. & $\mathrm{OH}$ & 2015 & FPH2015473 & I. verticillata $\times I$. serrata, Sparkleberry & MH171083 & MH171063 & MH171073 \\
\hline D. ilicicola sp. nov. & $\mathrm{OH}$ & 2015 & FPH $2015502 *$ & I. verticillata $\times I$. serrata, Bonfire & MH171084 & MH171064 & MH171074 \\
\hline D. ilicicola sp. nov. & $\mathrm{OH}$ & 2015 & FPH2015509 & I. verticillata $\times$ I. serrata, Sparkleberry & MH171085 & MH171065 & MH171075 \\
\hline D. ilicicola sp. nov. & $\mathrm{OH}$ & 2015 & FPH2015558 & I. verticillata $\times$ I. serrata, Sparkleberry & MH171086 & MH171066 & MH171076 \\
\hline D. ilicicola sp. nov. & $\mathrm{OH}$ & 2015 & FPH2015598 & I. verticillata $\times$ I. serrata, Sparkleberry & MH171087 & MH171067 & MH171077 \\
\hline D. ilicicola sp. nov. & $\mathrm{NC}$ & 2016 & FPH2016671 & Ilex sp. & MH171088 & MH171068 & MH171078 \\
\hline D. ilicicola sp. nov. & $\mathrm{OH}$ & 2016 & FPH2016702 & I. verticillata $\times$ I. serrata, Bonfire & MH171089 & MH171069 & MH171079 \\
\hline D. ilicicola sp. nov. & $\mathrm{OH}$ & 2016 & FPH2016706 & I. verticillata $\times$ I. serrata, Bonfire & MH171090 & MH171070 & MH171080 \\
\hline D. ilicicola sp. nov. & $\mathrm{OH}$ & 2016 & FPH2016725 & I. verticillata $\times I$. serrata, Bonfire & $\begin{array}{c}\text { MH171091 } \\
\text { ITS }\end{array}$ & MH171071 & MH171081 \\
\hline Epicoccum nigrum & $\mathrm{OH}$ & 2015 & FPH2015417* & I. verticillata $\times$ I. serrata, Bonfire & МH003637 & & \\
\hline E. nigrum & $\mathrm{OH}$ & 2015 & FPH2015505 & I. verticillata $\times$ I. serrata, Bonfire & MH003638 & & \\
\hline
\end{tabular}

${ }^{\mathrm{x}} \mathrm{MA}=$ Massachusetts; $\mathrm{NC}=$ North Carolina; $\mathrm{OH}=$ Ohio; PA = Pennsylvania.

y Isolates with an asterisk (*) were selected for use in pathogenicity experiments B and C.

${ }^{\mathrm{z}} \mathrm{EPG}=$ endopolygalacturonase; GAPDH = glyceraldehyde-3-phosphate dehydrogenase; HIS = histon H3; OPA1 and OPA10 = anonymous noncoding region; TUB $=\beta$-tubulin; ITS $=$ internal transcribed spacer. 
used for each isolate. Each fruit was wounded using a sterile needle and point inoculated using a micropipette with $8 \mu \mathrm{l}$ of a sterile waterTween 20 solution $(0.05 \% \mathrm{v} / \mathrm{v})$ containing $10^{5}$ conidia $/ \mathrm{ml}$ of each isolate. Ten wounded fruit inoculated with the same amount of sterile water-Tween 20 solution served as controls. All fruit were placed on a plastic tray in a completely randomized design and the entire tray was enclosed in a moist chamber made of a clear plastic bag containing a wet sterile paper towel. Moist chambers were incubated at $25^{\circ} \mathrm{C}$ for up to 4 weeks and fruit rot incidence was recorded for each isolate. Visual examination and identification of spores on symptomatic tissues as well as fungal reisolation and morphological identification of the isolates were performed to fulfill Koch's postulates.

One isolate per fungal species was selected from those used in experiment A. In two additional experiments (B and C), fruit were wounded, inoculated, and incubated as previously described. In both experiments, wounded and unwounded fruit inoculated with sterile water served as controls. Experiment B was conducted to test the effects of spore concentration and wounding on fruit infection. Experiments were carried out using a series of spore suspensions of each fungal species ranging from $10^{3}$ to $10^{6}$ conidia/ml. Inoculum was prepared by suspending spores from 7- to 21-day-old PDA cultures in sterile distilled water and by adjusting the concentration using a hemacytometer. Ten individual fruit per treatment were arranged in a complete randomized block design with six blocks. Fruit incidence and severity were recorded weekly for up to 6 weeks post inoculation. Finally, experiment $\mathrm{C}$ was conducted to test the effects of combined pathogen inoculum, spore concentration, and wounding on fruit infection. Spore suspensions at a concentration of $10^{2}, 10^{3}$, and $10^{5}$ conidia/ml were initially obtained for each fungal species. For each spore concentration, two pathogen species were combined $(1: 1 \mathrm{v} / \mathrm{v})$ to obtain the fungal treatment. All combinations of fungal species and spore concentrations were tested using six single fruit per treatment arranged in a complete randomized block design with six blocks. Disease evaluation was the same as previously described.

In all experiments, treatment effects were analyzed using PROC MIXED in SAS (version 9.4; SAS Institute Inc., Cary NC). Combined datasets from the two runs of each experiment were analyzed when results were in congruence. All data were arcsine transformed before statistical analyses were performed. Treatment means and 95\% confidence intervals were calculated in a transformed scale and back-transformed to present results. Least squares means
(LS-means; $\alpha=0.05$ ) were used for mean comparisons among treatments.

\section{Results}

Sample collection and fungal isolation. Three hundred and four symptomatic fruit samples out of the 623 processed resulted in the isolation of a total of 121 fungal isolates. Species of Diaporthe and Alternaria were the most frequently isolated fungi (39.47 and $32.89 \%$, respectively), followed by the genera Colletotrichum, Epicoccum Link, Cladosporium Link, Botryosphaeriaceae, Fusarium Link, and Phoma Sacc. (<10\%; Table 4). In a few cases, two fungi were isolated from the same lesion (Table 4). No fungal growth was observed from the remaining 319 samples.

Isolate characterization. For clarity of reporting, combined morphological and molecular characterization of the isolates is reported below.

Alternaria spp. Fourteen isolates tentatively identified as Alternaria sp. were assigned to four morphological groups based on colony and conidial morphology as well as sporulation patterns (Barnett and Hunter 1998). Ten isolates in the first group developed distinctive concentric rings of growth and sporulation. Isolates produced a primary chain of 3 to 11 conidia and abundant lateral secondary chains of 2 to 5 conidia. Conidia were brown, ellipsoid, with 3 to 6 transverse septa and sometimes one longitudinal septum. Characteristics were consistent with the description of Alternaria alternata (Fr.) Keissl. (Simmons 2007). Two isolates (FPH2015470 and FPH2015591) in the second group formed long chains of 4 to 9 conidia and occasionally secondary chains of 2 to 4 conidia. Conidia were wide ellipsoid or ovoid, constricted, with 4 to 7 transverse septa and rarely 1 longitudinal septum. Isolates in this group were tentatively identified as A. tenuissima (Nees) Wiltshire (Simmons 2007). One isolate (FPH2015338) in the third group was characterized by short primary chains of 4 to 7 conidia and occasionally secondary chains of 1 to 3 conidia. Conidia were wide ellipsoid or ovoid, constricted, and with 3 to 6 transverse septa and 1 to 2 longitudinal septa. These characteristics were consistent with the description of A. toxicogenica E. G. Simmons (Simmons 2007). One isolate (FPH2015395) in the fourth group produced long conidiophores and primary chains of 3 to 7 conidia and abundant secondary and tertiary chains of 1 to 3 conidia. Conidia were ellipsoid with 3 to 5 transverse septa and 0 to 2 longitudinal septa. This isolate was tentatively identified as A. arborescens E. G. Simmons (Simmons 2007). The

Table 2. Amplified loci, primers, PCR conditions, and references for each fungal genus characterized in this study

\begin{tabular}{|c|c|c|c|c|c|}
\hline \multirow[b]{2}{*}{ Fungal genus } & \multirow[b]{2}{*}{ Locus $^{\mathbf{y}}$} & \multirow[b]{2}{*}{ Primers (fw/rev) } & \multirow[b]{2}{*}{ PCR conditions } & \multicolumn{2}{|l|}{ References } \\
\hline & & & & Primers & $\mathbf{P C R}^{\mathrm{z}}$ \\
\hline \multirow[t]{3}{*}{ Alternaria } & endoPG & PG3/PG2b & $\begin{array}{l}95^{\circ} \mathrm{C} 1 \mathrm{~min} ;\left(95^{\circ} \mathrm{C} 30 \mathrm{~s}, 57^{\circ} \mathrm{C} 30 \mathrm{~s}, 72^{\circ} \mathrm{C} 30 \mathrm{~s}\right) \\
\times 35 \text { cycles; } 72^{\circ} \mathrm{C} 7 \mathrm{~min}\end{array}$ & Andrew et al. 2009 & Andrew et al. 2009 \\
\hline & OPA1 & OPA1-3L/OPA1-3Rb & $\begin{array}{l}94^{\circ} \mathrm{C} 1 \mathrm{~min} ;\left(94^{\circ} \mathrm{C} 20 \mathrm{~s}, 56^{\circ} \mathrm{C} 30 \mathrm{~s}, 72^{\circ} \mathrm{C} 40 \mathrm{~s}\right) \\
\times 35 \text { cycles; } 72^{\circ} \mathrm{C} 7 \mathrm{~min}\end{array}$ & Peever et al. 2004 & Peever et al. 2004 \\
\hline & OPA10 & OPA10-2L/OPA10-2R & $\begin{array}{l}95^{\circ} \mathrm{C} 1 \mathrm{~min} ;\left(95^{\circ} \mathrm{C} 30 \mathrm{~s}, 57^{\circ} \mathrm{C} 30 \mathrm{~s}, 72^{\circ} \mathrm{C} 30 \mathrm{~s}\right) \\
\times 35 \text { cycles; } 72^{\circ} \mathrm{C} 7 \mathrm{~min}\end{array}$ & Andrew et al. 2009 & Andrew et al. 2009 \\
\hline \multirow[t]{3}{*}{ Colletotrichum } & ITS & CaInt2/ITS4 & $\begin{array}{l}95^{\circ} \mathrm{C} 5 \mathrm{~min} ;\left(95^{\circ} \mathrm{C} 30 \mathrm{~s}, 60^{\circ} \mathrm{C} 30 \mathrm{~s}, 72^{\circ} \mathrm{C} 40 \mathrm{~s}\right) \\
\times 30 \text { cycles; } 72^{\circ} \mathrm{C} 5 \mathrm{~min}\end{array}$ & $\begin{array}{l}\text { McKay et al. 2009; } \\
\text { White et al. } 1990\end{array}$ & McKay et al. 2009 \\
\hline & TUB & $\mathrm{T} 1 / \mathrm{Bt}-2 \mathrm{~b}$ & $\begin{array}{l}95^{\circ} \mathrm{C} 4 \mathrm{~min} ;\left(95^{\circ} \mathrm{C} 30 \mathrm{~s}, 55^{\circ} \mathrm{C} 30 \mathrm{~s}, 72^{\circ} \mathrm{C} 45 \mathrm{~s}\right) \\
\times 35 \text { cycles; } 72^{\circ} \mathrm{C} 7 \mathrm{~min}\end{array}$ & $\begin{array}{l}\text { Glass and Donaldson 1995; } \\
\text { O’Donnell and Cigelnik } 1997\end{array}$ & Damm et al. 2012 \\
\hline & GAPDH & GDF/GDR & $\begin{array}{l}95^{\circ} \mathrm{C} 1 \mathrm{~min} ;\left(95^{\circ} \mathrm{C} 30 \mathrm{~s}, 57^{\circ} \mathrm{C} 30 \mathrm{~s}, 72^{\circ} \mathrm{C} 30 \mathrm{~s}\right) \\
\times 35 \text { cycles; } 72^{\circ} \mathrm{C} 7 \mathrm{~min}\end{array}$ & Templeton et al. 1992 & Damm et al. 2012 \\
\hline \multirow[t]{3}{*}{ Diaporthe } & ITS & ITS5/ITS4 & $\begin{array}{l}94^{\circ} \mathrm{C} 2 \mathrm{~min} ;\left(94^{\circ} \mathrm{C} 30 \mathrm{~s}, 58^{\circ} \mathrm{C} 60 \mathrm{~s}, 72^{\circ} \mathrm{C} 60 \mathrm{~s}\right) \\
\times 40 \text { cycles; } 72^{\circ} \mathrm{C} 3 \mathrm{~min}\end{array}$ & White et al. 1990 & Gomes et al. 2013 \\
\hline & HIS & CYLH3F/H3-1b & $\begin{array}{l}95^{\circ} \mathrm{C} 1 \mathrm{~min} ;\left(95^{\circ} \mathrm{C} 30 \mathrm{~s}, 62^{\circ} \mathrm{C} 60 \mathrm{~s}, 72^{\circ} \mathrm{C} 60 \mathrm{~s}\right) \\
\times 40 \text { cycles; } 72^{\circ} \mathrm{C} 3 \mathrm{~min}\end{array}$ & $\begin{array}{l}\text { Crous et al. 2004; } \\
\text { Glass and Donaldson } 1995\end{array}$ & Gomes et al. 2013 \\
\hline & TUB & T1/CYLTUB1R & $\begin{array}{l}95^{\circ} \mathrm{C} 1 \mathrm{~min} ;\left(95^{\circ} \mathrm{C} 30 \mathrm{~s}, 60^{\circ} \mathrm{C} 60 \mathrm{~s}, 72^{\circ} \mathrm{C} 60 \mathrm{~s}\right) \\
\times 40 \text { cycles; } 72^{\circ} \mathrm{C} 3 \mathrm{~min}\end{array}$ & $\begin{array}{l}\text { Crous et al. 2004; } \\
\text { O’Donnell and Cigelnik } 1997\end{array}$ & Gomes et al. 2013 \\
\hline Epicoccum & ITS & ITS1/ITS4 & $\begin{array}{l}94^{\circ} \mathrm{C} 5 \mathrm{~min} ;\left(94^{\circ} \mathrm{C} 45 \mathrm{~s}, 57^{\circ} \mathrm{C} 45 \mathrm{~s}, 72^{\circ} \mathrm{C} 90 \mathrm{~s}\right) \\
\times 35 \text { cycles; } 72^{\circ} \mathrm{C} 10 \mathrm{~min}\end{array}$ & White et al. 1990 & White et al. 1990 \\
\hline
\end{tabular}

y endoPG = endopolygalacturonase, GAPDH = glyceraldehyde-3-phosphate dehydrogenase, $\mathrm{HIS}=$ histon H3, OPA1 and OPA10 = anonymous noncoding region, TUB $=\beta$-tubulin, ITS $=$ internal transcribed spacer.

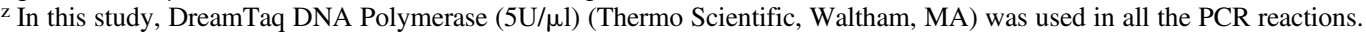


Table 3. Reference sequences for Alternaria, Colletotrichum, Diaporthe, and Epicoccum retrieved from GenBank that were included in the phylogenetic analyses

\begin{tabular}{|c|c|c|c|c|c|c|}
\hline \multirow[b]{2}{*}{ Species } & \multirow[b]{2}{*}{ Strain ${ }^{y}$} & \multirow[b]{2}{*}{ Origin } & \multirow[b]{2}{*}{ Host } & \multicolumn{3}{|c|}{ GenBank accession no. ${ }^{\mathrm{z}}$} \\
\hline & & & & endoPG & OPA1-3 & OPA10-2 \\
\hline Alternaria alternata & CBS 102596 & USA & Citrus jambhiri & KP124030 & AY295047 & KP124637 \\
\hline A. alternata & CBS 102598 & USA & Citrus jambhiri & AY295026 & AY295048 & KP124638 \\
\hline A. alternata & CBS 102600 & USA & Citrus reticulata & KP124033 & AY295042 & KP124640 \\
\hline A. alternata & CBS 102602 & Turkey & Minneola tangelo & AY295023 & AY295052 & KP124641 \\
\hline A. alternata & CBS 102604 & Israel & Minneola tangelo & KP124035 & AY631443 & KP124643 \\
\hline A. alternata & CBS 104.26 & Unknown & Unknown & KP123995 & - & KP124603 \\
\hline A. alternata & CBS 106.24 & USA & Malus sylvestris & AY295020 & JQ800572 & JQ800620 \\
\hline A. alternata & CBS 115152 & China & Psychotria serpens & KP124049 & - & KP124658 \\
\hline A. alternata & CBS 117143 & Italy & Capsicum anпиит & KP124055 & - & KP124665 \\
\hline A. alternata & CBS 911.97 & India & Artemisia brevifolia & KP124027 & - & KP124634 \\
\hline A. alternata & CBS 916.96 & India & Arachis hypogaea & JQ811978 & AY295043 & KP124632 \\
\hline A. alternata & CBS 918.96 & UK & Dianthus chinensis & KP124026 & JQ859820 & KP124633 \\
\hline A. alternata & CBS 966.95 & India & Solanum lycopersicum & KP124024 & - & KP124630 \\
\hline A. alternata & EGS 44-159 & Turkey & Citrus reticulata $\times C$. paradisi & AY295022 & AY295051 & EF504051 \\
\hline A. arborescens & CBS 102605 & USA & Solanum lycopersicum & AY295028 & AY295035 & KP124712 \\
\hline A. betae-kenyensis & CBS 118810 & Kenya & Beta vulgaris & KP124123 & - & KP124733 \\
\hline A. eichhorniae & CBS 489.92 & India & Eichhornia crassipes & $\begin{array}{c}\text { KP124130 } \\
\text { GAPDH }\end{array}$ & $\stackrel{-}{\text { TUB }}$ & KP124740 \\
\hline Colletotrichum acutatum & CBS 112996 & Australia & Canica papaya & JQ948677 & JQ005860 & \\
\hline C. cosmi & CBS 853.73 & Netherlands & Cosmos sp. & JQ948604 & JQ949925 & \\
\hline C. fioriniae & CBS 128517 & USA & Fiorinia externa & JQ948622 & JQ949943 & \\
\hline C. fioriniae & CBS 200.35 & USA & Rubus sp. & JQ948623 & JQ949944 & \\
\hline C. fioriniae & CBS 293.67 & Australia & Persea americana & JQ948640 & JQ949961 & \\
\hline C. guajavae & IMI 350839 & India & Psidium guajava & JQ948600 & JQ949921 & \\
\hline C. nymphaeae & CBS 515.78 & Netherlands & Nymphaea alba & JQ948527 & JQ949848 & \\
\hline C. scovillei & CBS 126529 & Indonesia & Capsicum sp. & JQ948597 & JQ949918 & \\
\hline C. walleri & CBS 125472 & Colombia & Coffea sp. & JQ948605 & JQ949926 & \\
\hline C. johnstonii & CBS 128532 & New Zealand & Solanum lycopersicum & $\begin{array}{c}\text { JQ948775 } \\
\text { HIS }\end{array}$ & $\begin{array}{c}\text { JQ950095 } \\
\text { ITS }\end{array}$ & TUB \\
\hline Diaporthe acaciigena & CBS 129521 & Australia & Acacia retinodes & KC343489 & KC343005 & KC343973 \\
\hline D. acerina & CBS 137.27 & - & Acer saccharum & KC343490 & KC343006 & KC343974 \\
\hline D. alleghaniensis & CBS 495.72 & Canada & Betula alleghaniensis & KC343491 & KC343007 & KC343975 \\
\hline D. alnea & CBS 146.46 & Netherlands & Alnus sp. & KC343492 & KC343008 & KC343976 \\
\hline D. ampelina & CBS 114016 & France & Vitis vinifera & - & AF230751 & $\mathrm{JX} 275452$ \\
\hline D. amygdali & CBS 126679 & Portugal & Prunus dulcis & KC343506 & KC343022 & KC343990 \\
\hline D. apiculata & CGMCC 3.17533 & China & Camellia sinensis & - & KP267896 & KP293476 \\
\hline D. australafricana & CBS 111886 & Australia & Vitis vinifera & KC343522 & KC343038 & KC344006 \\
\hline D. beckhausii & CBS 138.27 & - & Viburnum sp. & KC343525 & KC343041 & KC344009 \\
\hline D. betulae & CFCC 50469 & China & Betula platyphylla & KT732999 & KT732950 & KT733020 \\
\hline D. betulicola & CFCC 51128 & China & Betula albosinensis & KX024661 & KX024653 & KX024657 \\
\hline D. bicincta & CBS 121004 & USA & Juglans sp. & KC343618 & KC343134 & KC344102 \\
\hline D. biguttusis & CGMCC 3.17081 & China & Lithocarpus glabra & - & KF576282 & KF576306 \\
\hline D. brasiliensis & CBS 133183 & Brazil & Aspidosperma tomentosum & KC343526 & KC343042 & KC 344010 \\
\hline D. carpini & CBS 114437 & Sweden & Carpinus betulus & KC343528 & KC343044 & KC344012 \\
\hline D. caulivora & CBS 127268 & Croatia & Glycine max & KC343529 & KC343045 & KC344013 \\
\hline D. celastrina & CBS 139.27 & USA & Celastrus scandens & KC343531 & KC343047 & KC344015 \\
\hline D. cf. heveae 1 & CBS 852.97 & Brazil & Hevea brasiliensis & KC343600 & KC343116 & KC344084 \\
\hline D. cf. heveae 2 & CBS 681.84 & India & Hevea brasiliensis & KC343601 & KC343117 & KC344085 \\
\hline D. cf. nobilis & CBS 587.79 & Japan & Pinus pentaphylla & KC343637 & KC343153 & KC344121 \\
\hline D. charlesworthii & BRIP 54884m & Australia & Rapistrum rugosum & - & KJ197288 & KJ197268 \\
\hline D. citri & CBS 135422 & USA & Citrus sp. & KJ420881 & NR145304 & KC843187 \\
\hline D. citrichinensis & ZJUD34 & China & Citrus unshiu & KJ490516 & JQ954648 & KJ490396 \\
\hline D. convolvuli & CBS 124654 & Turkey & Convolvulus arvensis & KC343538 & KC343054 & KC344022 \\
\hline D. crataegi & CBS 114435 & Sweden & Crataegus oxyacantha & KC343539 & KC343055 & KC344023 \\
\hline D. cynaroidis & CBS 122676 & South Africa & Protea cynaroides & KC343542 & KC343058 & KC344026 \\
\hline D. decedens & CBS 109772 & Austria & Corylus avellana & KC343543 & KC343059 & KC344027 \\
\hline D. detrusa & CBS 109770 & Austria & Berberis vulgaris & KC343545 & KC343061 & KC344029 \\
\hline D. ellipicola & CGMCC 3.17084 & China & Lithocarpus glabra & - & KF576270 & KF576294 \\
\hline & & & & & (Continue & n next page) \\
\hline
\end{tabular}

y Ex-holotype, ex-epitype, ex-type, authentic, or representative cultures are in bold. AR = Collection of Systematic Mycology and Microbiology Laboratory, USDA-ARS, Beltsville; ATCC = American Type Culture Collection; BRIP = Plant Pathology Herbarium, Dutton Park, Queensland, Australia; CBS: Centraalbureau voor Schimmelcultures, Fungal Biodiversity Centre, Utrecht, The Netherlands; CFCC = China Forestry Culture Collection Center, Beijing, China; CGMCC = China General Microbial Culture Collection Center, China; CMW = Forestry and Agricultural Biotechnology Institute, University of Pretoria, South Africa; EGS = Collection of E.G. Simmons; IMI: Culture collection of CABI Europe UK Centre, Egham, UK; LC: Collection of L. Cai; PD: Plant Protection Service, Wageningen, the Netherlands; ZJUD = Zhejiang University, China.

${ }^{\mathrm{z}}$ endoPG = endopolygalacturonase; GAPDH = glyceraldehyde-3-phosphate dehydrogenase; HIS = histon H3; OPA1-3 and OPA10-2 = anonymous noncoding region; TUB $=\beta$-tubulin; ITS $=$ internal transcribed spacer 
Table 3. (Continued from previous page)

\begin{tabular}{|c|c|c|c|c|c|c|}
\hline \multirow[b]{2}{*}{ Species } & \multirow[b]{2}{*}{ Strain $^{y}$} & \multirow[b]{2}{*}{ Origin } & \multirow[b]{2}{*}{ Host } & \multicolumn{3}{|c|}{ GenBank accession no. ${ }^{\mathrm{z}}$} \\
\hline & & & & endoPG & OPA1-3 & OPA10-2 \\
\hline D. eres & CBS 101742 & Netherlands & Fraxinus sp. & KC343557 & KC343073 & KC344041 \\
\hline D. eres & CBS 109767 & Austria & Acer campestre & KC343559 & KC343075 & KC344043 \\
\hline D. eres & CBS 122.82 & Netherlands & Skimmia japonica & KC343561 & KC343077 & KC344045 \\
\hline D. eres & CBS 129168 & Latvia & Rhododendron sp. & KC343562 & KC343078 & KC344046 \\
\hline D. eres & CBS 138594 & Germany & Ulmus laevis & KJ420850 & KJ210529 & KJ420799 \\
\hline D. eres & CBS 186.37 & UK & Picea abies & KC343563 & KC343079 & KC344047 \\
\hline D. eres & CBS 250.38 & UK & Fraxinus excelsior & KC343564 & KC343080 & KC344048 \\
\hline D. eres & CBS 267.32 & - & - & KC343565 & KC343081 & KC344049 \\
\hline D. eres & CBS 267.55 & Netherlands & Laburmum $\times$ watereri & KC343566 & KC343082 & KC344050 \\
\hline D. eres & CBS 283.85 & Netherlands & Allium giganteum & KC343567 & KC343083 & KC344051 \\
\hline D. eres & CBS 287.74 & Netherlands & Sorbus aucuparia & KC343568 & KC343084 & KC344052 \\
\hline D. eres & CBS 297.77 & Netherlands & Osmanthus aquifolium & KC343569 & KC343085 & KC344053 \\
\hline D. eres & CBS 365.97 & Netherlands & Opuntia sp. & KC343570 & KC343086 & KC344054 \\
\hline D. eres & CBS 370.67 & Netherlands & Ilex aquifolium & KC343571 & KC343087 & KC344055 \\
\hline D. eres & CBS 375.61 & - & Malus sylvestris & $\mathrm{KC} 343572$ & KC343088 & KC344056 \\
\hline D. eres & CBS 422.50 & Netherlands & Phaseolus vulgaris & KC343573 & KC343089 & KC344057 \\
\hline D. eres & CBS 439.82 & UK & Cotoneaster sp. & KC343574 & KC343090 & KC344058 \\
\hline D. eres & CBS 445.62 & Netherlands & Alliaria officinalis & KC343575 & KC343091 & KC344059 \\
\hline D. eres & CBS 485.96 & Netherlands & Rumex hydrolapathum & KC343576 & KC343092 & KC344060 \\
\hline D. eres & CBS 528.83 & Netherlands & Wisteria sinensis & KC343577 & KC343093 & KC344061 \\
\hline D. eres & CBS 688.97 & Netherlands & Abutilon sp. & KC343578 & KC343094 & KC344062 \\
\hline D. eres & CBS 694.94 & Netherlands & Ilex aquifolium & KC343579 & KC343095 & KC344063 \\
\hline D. eres & CBS 791.68 & Netherlands & Magnolia $\times$ soulangeana & KC343580 & KC343096 & KC344064 \\
\hline D. eres & CBS 841.84 & Netherlands & Hordeum sp. & KC343581 & KC343097 & KC344065 \\
\hline D. fibrosa & CBS 109751 & Austria & Rhamnus cathartica & KC343583 & KC343099 & KC344067 \\
\hline D. foeniculina & CBS 187.27 & Italy & Camellia sinensis & KC343591 & KC343107 & KC344075 \\
\hline D. fraxini-angustifoliae & BRIP 54781 & Australia & Fraxinus angustifolia & - & JX862528 & KF170920 \\
\hline D. gardeniae & CBS 288.56 & Italy & Gardenia florida & KC343597 & KC343113 & KC344081 \\
\hline D. goulteri & BRIP 55657a & Australia & Helianthus annuиs & - & KJ197290 & KJ197270 \\
\hline D. helianthi & CBS 592.81 & Serbia & Helianthus annииs & KC343599 & KC343115 & KC344083 \\
\hline D. helicis & AR 5211 & France & Hedera helix & KJ420875 & KJ210538 & KJ420828 \\
\hline D. hickoriae & CBS 145.26 & USA & Carya glabra & KC343602 & KC343118 & KC344086 \\
\hline D. hongkongensis & CBS 115448 & China & Dichroa febrifuga & KC343603 & KC343119 & KC344087 \\
\hline D. impulsa & CBS 114434 & Sweden & Sorbus aucuparia & KC343605 & KC343121 & KC344089 \\
\hline D. juglandicola & CFCC 51134 & China & Juglans mandshurica & KX024622 & KU985101 & KX024634 \\
\hline D. longicicola & CGMCC 3.17089 & China & Lithocarpus glabra & - & KF576267 & KF576291 \\
\hline D. longicolla & ATCC 60325 & USA & Glycine $\max$ & KJ659188 & NR144924 & KJ610883 \\
\hline D. longispora & CBS 194.36 & Canada & Ribes sp. & KC343619 & KC343135 & KC344103 \\
\hline D. lusitanicae & CBS 123212 & Portugal & Foeniculum vulgare & KC343620 & KC343136 & KC344104 \\
\hline D. macintoshii & BRIP 55064a & Australia & Rapistrum rugosum & - & KJ197289 & KJ197269 \\
\hline D. mahothocarpus & CGMCC 3.15181 & China & Lithocarpus glabra & - & KC153096 & KF576312 \\
\hline D. manihotia & CBS 505.76 & Rwanda & Manihot utilizsima & KC343622 & KC343138 & KC344106 \\
\hline D. masirevicii & BRIP 54256 & Australia & Glycine max & - & KJ197277 & KJ197257 \\
\hline D. mayteni & CBS 133185 & Brazil & Maytenus ilicifolia & KC343623 & KC343139 & KC344107 \\
\hline D. melonis & CBS 507.78 & USA & Cucumis melo & KC343626 & KC343142 & KC344110 \\
\hline D. miriciae & BRIP 54736j & Australia & Helianthus anпuиs & - & KJ197282 & KJ197262 \\
\hline D. musigena & CBS 129519 & Australia & Musa sp. & KC343627 & KC343143 & KC344111 \\
\hline D. neilliae & CBS 144.27 & USA & Spiraea sp. & KC343628 & KC343144 & KC344112 \\
\hline D. neoarctii & CBS 109490 & USA & Ambrosia trifida & KC343629 & KC343145 & KC344113 \\
\hline D. nomurai & CBS 157.29 & Japan & Moru sp. & KC343638 & KC343154 & KC344122 \\
\hline D. nothofagi & BRIP 54801 & Australia & Nothofagus cunninghamii & - & JX862530 & KF170922 \\
\hline D. novem & CBS 127270 & Croatia & Glycine max & KC343640 & KC343156 & KC344124 \\
\hline D. ocoteae & CBS 141330 & France & Ocotea obtusata & - & KX228293 & KX228388 \\
\hline D. oncostroma & CBS 809.85 & Germany & Ilex aquifolium & KC343647 & KC343163 & KC344131 \\
\hline D. oxe & CBS 133186 & Brazil & Maytenus ilicifolia & KC343648 & KC343164 & KC344132 \\
\hline D. padi & CBS 114200 & Sweden & Prunus padus & KC343653 & KC343169 & KC344137 \\
\hline D. paranensis & CBS 133184 & Brazil & Maytenus ilicifolia & KC343655 & KC343171 & KC344139 \\
\hline D. penetriteum & CGMCC 3.17532 & China & Camellia sinensis & KP714493 & KP267879 & KP293459 \\
\hline D. perjuncta & CBS 109745 & Austria & Ulmus glabra & KC343656 & KC343172 & KC344140 \\
\hline D. phaseolorum & AR 4203 & USA & Phaseolus vulgaris & KJ659220 & KJ590738 & KJ610893 \\
\hline D. pseudomangiferae & CBS 101339 & Dominican & Mangifera indica & KC343665 & KC343181 & KC344149 \\
\hline D. pulla & CBS 338.89 & Yugoslavia & Hedera helix & KC343636 & KC343152 & KC344120 \\
\hline D. pustulata & CBS 109742 & Austria & Acer pseudoplatanus & KC343669 & KC343185 & KC344153 \\
\hline D. pustulata & CBS 109760 & Austria & Acer pseudoplatanus & KC343670 & KC343186 & KC344154 \\
\hline D. pustulata & CBS 109784 & Austria & Prunus padus & KC343671 & KC343187 & KC344155 \\
\hline & & & & & (Continu & n next page) \\
\hline
\end{tabular}


Table 3. (Continued from previous page)

\begin{tabular}{|c|c|c|c|c|c|c|}
\hline \multirow[b]{2}{*}{ Species } & \multirow[b]{2}{*}{ Strain $^{y}$} & \multirow[b]{2}{*}{ Origin } & \multirow[b]{2}{*}{ Host } & \multicolumn{3}{|c|}{ GenBank accession no. ${ }^{\mathrm{z}}$} \\
\hline & & & & endoPG & OPA1-3 & OPA10-2 \\
\hline D. raonikayaporum & CBS 133182 & Brazil & Spondias mombin & KC343672 & KC343188 & KC344156 \\
\hline D. rudis & CBS 113201 & Portugal & Vitis vinifera & KC343718 & KC343234 & KC344202 \\
\hline D. sackstonii & BRIP 54669b & Australia & Helianthus annuus & - & KJ197287 & KJ197267 \\
\hline D. salicicola & BRIP 54825 & Australia & Salix purpurea & - & JX862531 & KF170923 \\
\hline D. schini & CBS 133181 & Brazil & Schinus terebinthifolius & KC343675 & KC343191 & KC344159 \\
\hline D. scobina & CBS 251.38 & UK & Fraxinus excelsior & KC343679 & KC343195 & KC344163 \\
\hline D. subclavata & ZJUD95 & China & Citrus sp. & KJ490572 & KJ490630 & KJ490451 \\
\hline D. terebinthifolii & CBS 133180 & Brazil & Schinus terebinthifolius & KC343700 & KC343216 & KC344184 \\
\hline D. toxica & CBS 534.93 & Australia & Lupinus angustifolius & KC343704 & KC343220 & KC344188 \\
\hline D. vaccinii & CBS 160.32 & USA & Oxycoccus macrocarpos & KC343712 & KC343228 & KC344196 \\
\hline D. vawdreyi & BRIP 57887a & Australia & Psidium guajava & - & KR936126 & KR936128 \\
\hline D. virgiliae & CMW 40755 & South Africa & Virgilia oroboides & - & KP247573 & KP247583 \\
\hline D. wollworthii & CBS 148.27 & - & Ulmus americana & KC343729 & KC343245 & KC344213 \\
\hline Diaporthella corylina & CBS 121124 & China & Corylus sp. & $\begin{array}{c}\mathrm{KC} 343488 \\
\text { ITS }\end{array}$ & KC343004 & KC343972 \\
\hline Epicoccum brasiliense & CBS 120105 & Brazil & Amaranthus Sp. & GU237760 & & \\
\hline E. camelliae & CGMCC 3.18343 & China & Camellia sinensis & KY742091 & & \\
\hline E. keratinophilum & CBS 142455 & USA & Human superficial tissue & LT592930 & & \\
\hline E. latusicollum & CGMCC 3.18346 & China & Sorghum bicolor & KY742101 & & \\
\hline E. nigrum & CBS 125.82 & Netherlands & Human toe nail & FJ426995 & & \\
\hline E. nigrum & CBS 173.73 & USA & Dactylis glomerata & FJ426996 & & \\
\hline E. nigrum & LC 8158 & USA & Роа аппиа & KY742111 & & \\
\hline E. nigrum & LC 8159 & USA & Роа аппиа & KY742112 & & \\
\hline E. ovisporum & CBS 180.80 & South Africa & Zea mays & FJ427068 & & \\
\hline E. pimprinum & CBS 246.60 & India & Soil & KY742113 & & \\
\hline E. pimprinum & PD $77 / 1028$ & India & Soil & KY742114 & & \\
\hline
\end{tabular}

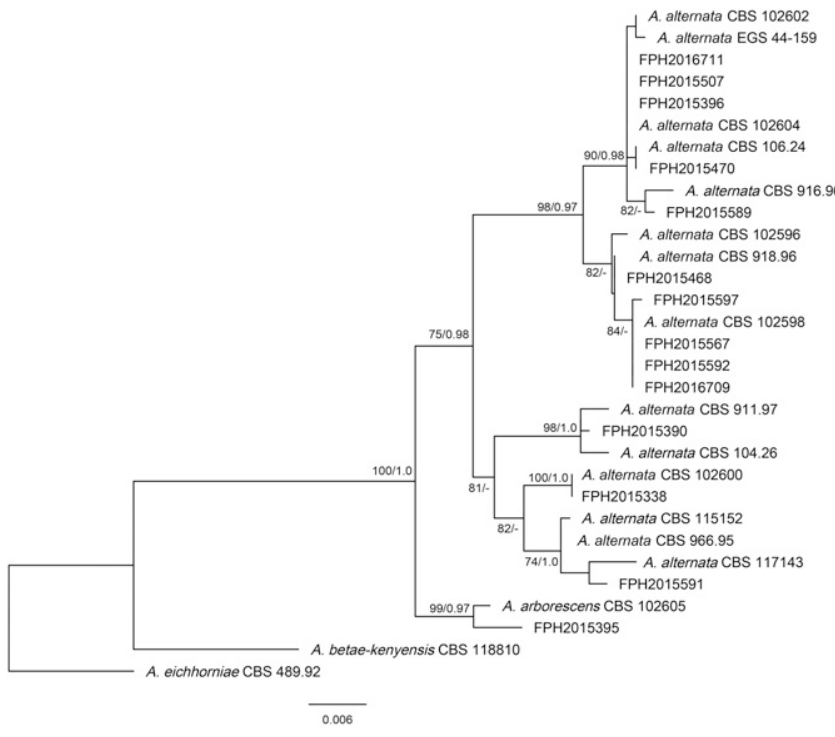

Fig. 2. Maximum likelihood (ML) phylogenetic tree based on the Kimura 2-parameter plus a gamma distribution model (Kimura 1980) obtained from combined endoPG, OPA1, and OPA10 datasets of Alternaria spp. The ML bootstrap support (BS) $\geq 70$ and Bayesian posterior probability (PP) $\geq 0.95$ values are indicated at the nodes (BS/PP). The tree was rooted with A. eichhorniae (CBS 489.92).

concatenated datasets of three loci (endoPG, OPA1, and OPA10) were analyzed using ML and BI and similar tree topologies were obtained. Thirty-one sequences were included in the phylogenetic analysis consisting of 17 reference strains (Table 3; Andrew et al. 2009; Peever et al. 2004; Rotondo et al. 2012; Woudenberg et al. 2015) and 14 isolates from this study. Isolate FPH2015395 grouped with $A$. arborescens reference strain CBS 102605 with a high bootstrap support value $(\mathrm{BS}=96)$ and a high Bayesian posterior probability $(\mathrm{PP}=0.97)$. All other isolates clustered with several A. alternata reference sequences $(\mathrm{BS}=75, \mathrm{PP}=0.96$; Fig. 2).
Diaporthe spp. In total, 13 isolates were identified as Diaporthe spp. Of these, three isolates (FPH201322, FPH2015394, and FPH2017229) were characterized by white, fluffy, aerial mycelium, gray to brown on the reverse side. Pycnidia in culture were dark brown to black, globose or subglobose, erumpent, solitary or aggregated, arranged in concentric rings. Creamy to yellow conidial masses were globose or exuded in cirrhi. Alpha conidia were hyaline, aseptate, smooth, guttulate, and ellipsoid. Beta conidia were hyaline, aseptate, slightly curved, hamate or filiform, and less common than alpha conidia. Even though these three isolates had similar morphological characteristics, the width of both alpha and beta conidia of isolate FPH2017229 was significantly smaller (Table 5; significance reference not shown). All three isolates were tentatively assigned to the Diaporthe eres Nitschke species complex (Baumgartner et al. 2013; Dissanayake et al. 2015; Udayanga et al. 2014a). The 10 remaining isolates presented morphological characteristics that differed from the three isolates previously described as well as from other Diaporthe species described in the literature, including those previously reported from Ilex spp. (Dissanayake et al. 2017; Du et al. 2016; Gomes et al. 2013; Huang et al. 2013, 2015; Machingambi et al. 2015; Mostert et al. 2001; Tan et al. 2013; Thompson et al. 2015; Udayanga et al. 2015; Wehmeyer 1933; Yang et al. 2017). Detailed morphological features of these isolates are reported in the new species description below (Fig. 3).

Two sets of multilocus phylogenetic analyses were conducted for Diaporthe spp. isolates. In the first analysis, the concatenated HIS, ITS, and TUB datasets consisted of 102 isolates from 82 taxa (Dissanayake et al. 2017; Du et al. 2016; Gomes et al. 2013; Huang et al. 2013, 2015; Machingambi et al. 2015; Mostert et al. 2001; Tan et al. 2013; Thompson et al. 2015; Udayanga et al. 2012, 2014a, b, 2015; Yang et al. 2017). In both ML and BI analyses, the 13 isolates from this study grouped into three different clades (Fig. 4). Three isolates (FPH201322, FPH2015394, and FPH2017229), in accordance with their morphology, clustered within the D. eres species complex, and grouped with reference strains CBS 138594, CBS 287.74, CBS 370.67, and CBS 587.79. The remaining 10 isolates clustered with strong support $(\mathrm{BS}=85$ and $\mathrm{PP}=1)$ as a unique and unnamed clade sharing a strongly supported phylogenetic 
relationship (BS $=99$ and $\mathrm{PP}=1)$ near the reference strain of D. $c f$. heveae 1 Petch (CBS 852.97; Fig. 4). Morphological and molecular data revealed that these 10 isolates represented a unique and undescribed species of Diaporthe with no apparent species name. Thus, we hereby propose a new species name to properly identify this unique taxon:

D. ilicicola S. Lin, Taylor \& Peduto Hand, sp. nov.

MycoBank No. MB824842

Etymology - named according to the host it was first isolated from, namely Ilex verticillata $\times$ I. serrata.

Conidiomata pycnidial, dark brown to black, erumpent, solitary or aggregated, ostiolate, creamy to light salmon conidial mass globose or exuding in cirrhi, arranged in circle at the edge of colony. Conidiophores hyaline, subcylindrical, reduced to conidiogenous cells. Conidiogenous cells hyaline, subcylindrical, tapering toward the apex, 7.64 to $14.14 \times 1.9$ to $3.82 \mu \mathrm{m}$ (av. $\pm \mathrm{SD}=11.2 \pm 0.4 \times$ $2.7 \pm 0.14 \mu \mathrm{m})$. Alpha conidia hyaline, aseptate, smooth, ovate to
Table 4. Isolation frequency of the different fungi recovered from the 304 symptomatic holly fruit that yielded fungal growth between 2013 and 2017 across all sampling locations

\begin{tabular}{lc}
\hline Fungal species & Isolation frequency (\%) \\
\hline Diaporthe spp. & 39.47 \\
Alternaria spp. & 32.89 \\
Colletotrichum spp. & 7.24 \\
Epicoccum spp. & 4.61 \\
Cladosporium spp. & 3.68 \\
Alternaria sp. + Diaporthe sp. & 2.34 \\
Botryosphaeriaceae & 2.01 \\
Alternaria sp. + Colletotrichum sp. & 1.67 \\
Fusarium spp. & 1.00 \\
Phoma spp. & 1.00 \\
Epicoccum sp. + Diaporthe sp. & 1.00 \\
Alternaria sp. + Fusarium sp. & 0.33 \\
\hline
\end{tabular}
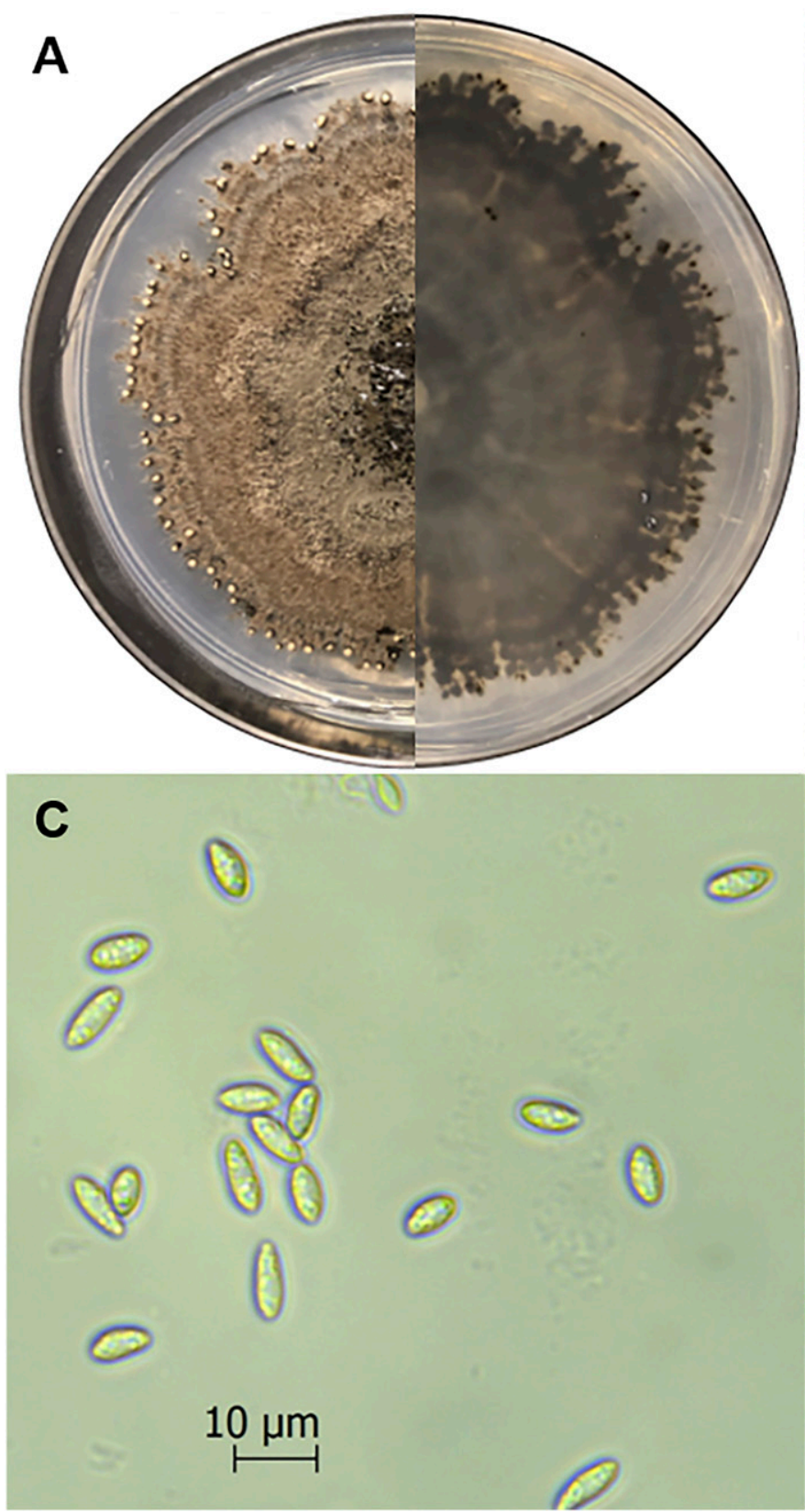

Fig. 3. Morphology of Diaporthe ilicicola sp. nov. strain FPH2015502. A, colony on PDA after 28 days at $23^{\circ} \mathrm{C}$; top and bottom of the plate; $\mathrm{B}$, conidiogenous cells and alpha conidia; C, alpha conidia; D, sporulation from conidiomata on PDA.

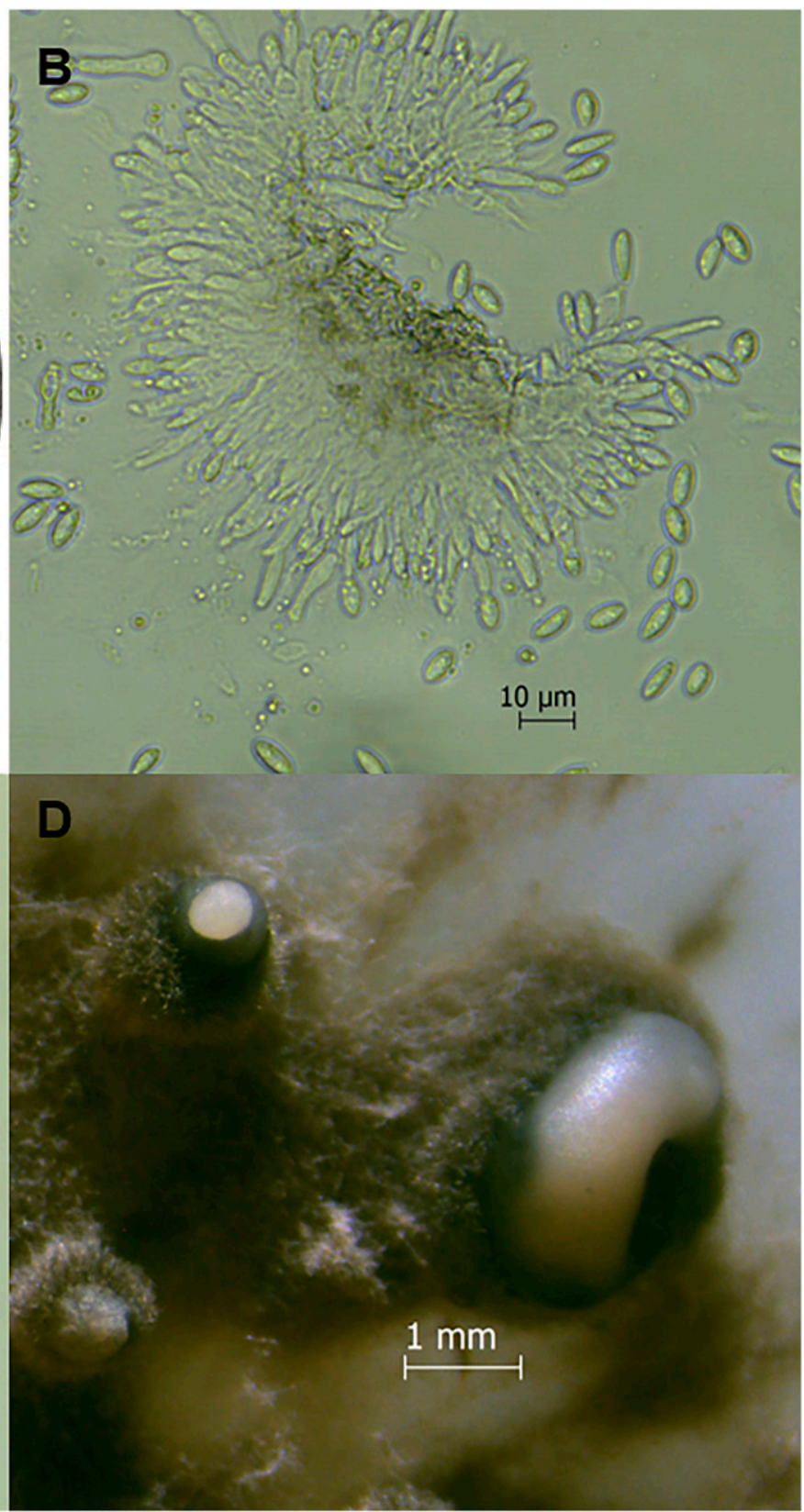


ellipsoid, guttulate, on PDA 7.13 to $9.43 \times 2.91$ to $4.16 \mu \mathrm{m}$ (av. $\pm \mathrm{SD}=8.41 \pm 0.1 \times 3.63 \pm 0.05 \mu \mathrm{m})$. Beta conidia not observed. Sexual morph not observed.

Culture characteristics - Colonies on PDA at $23^{\circ} \mathrm{C}$ with 12 -h photoperiod slow growing, $2.89 \pm 0.08 \mathrm{~mm} / \mathrm{day}$, flat and compact, irregular edge, originally white to olive, turning to grayish brown while aging, reverse dark brown.

Host - I. verticillata $\times I$. serrata

Distribution - United States

Specimens examined. United States, Ohio, Madison, on rotten fruit of I. verticillata $\times$ I. serrata, cultivar Bonfire, December 2015, Shan Lin, holotype FPH2015502 (CBS 144318).

In the second analysis, the concatenated HIS, ITS, and TUB datasets consisted of 39 isolates from 16 taxa that are within or closely related to the D. eres species complex (Gao et al. 2016; Gomes et al. 2013; Udayanga et al. 2014a) and three Diaporthe isolates from this study (FPH201322, FPH2015392, and FPH2017229). According to the new analysis, isolates FPH201322 and FPH2015392 grouped with D. eres reference strains $(\mathrm{BS}=99, \mathrm{PP}=1.0$ and $\mathrm{BS}=97, \mathrm{PP}=$ 0.95 , respectively), while isolate FPH2017229 grouped with a reference strain of $D$. cf. nobilis (CBS 587.79; $\mathrm{BS}=90, \mathrm{PP}=0.99$; Fig. 5).

Colletotrichum spp. Eight isolates tentatively identified as Colletotrichum spp. were divided into two morphological groups. All but one isolate belonged to the first group and were characterized by cottony mycelium that was light gray to gray on the upper side of the plate, and brownish pink with abundant scattered melanized spots on the reverse side. Orange conidial masses formed in the center of the colony after 21 days. Conidia were hyaline, aseptate, fusiform to cylindrical, and slightly pointed at one or both ends. These features were consistent with the description of Colletotrichum fioriniae (Marcelino \& Gouli) Pennycook (Shivas and Tan 2009). The isolate in the second morphological group (FPH2015590) presented white to gray colored mycelium on the upper side of the plate and salmon on the reverse side. Conidia were hyaline, aseptate, fusiform to cylindrical, with one or both ends slightly acute. This isolate was identified as C. nymphaeae (Pass.) Aa (Velho et al. 2015). DNA from all Colletotrichum isolates was successfully amplified using $C$. acutatum species complex-specific primers targeting the ITS region, and sequence analysis confirmed that they belonged to the $C$. acutatum species complex (McKay et al. 2009). In the multigene phylogenetic analyses of combined GAPDH and TUB datasets using ML and BI, isolates grouped into two clades. Isolate FPH2015590 clustered with C. nymphaeae strain CBS 515.78, while the rest of the isolates clustered with $C$. fioriniae strains CBS 128517, CBS 200.35, and CBS 293.67 (Damm et al. 2012; Fig. 6).

Epicoccum spp. Two isolates tentatively identified as Epicoccum $\mathrm{sp}$. were characterized by yellow to orange colonies producing an

Table 5. Conidial measurements and mycelial growth rate of select isolates of Alternaria, Colletotrichum, Diaporthe, and Epicoccum retrieved from symptomatic winterberry fruit used in this study (Diaporthe ilicicola sp. nov. data not included)

\begin{tabular}{|c|c|c|c|c|}
\hline Morphospecies & Isolate & Conidium length $(\mu \mathrm{m})$ & Conidium width $(\mu \mathrm{m})$ & Mycelial growth rate (mm/day) \\
\hline \multirow[t]{11}{*}{$\overline{\text { Alternaria alternata }}$} & FPH2015390 & $32.58 \pm 0.63(27.86-42.8)^{\mathrm{v}}$ & $11.09 \pm 0.26(8.79-13.9)$ & $5.39 \pm 0.06(5.31-5.54)^{\mathrm{w}}$ \\
\hline & FPH2015396 & $31.85 \pm 0.67(31.49-40)$ & $9.39 \pm 0.24(6.93-12.28)$ & $5.4 \pm 0.04(5.3-5.49)$ \\
\hline & FPH2015468 & $30.85 \pm 0.92(23.84-39.71)$ & $11.11 \pm 0.3(8.29-13.75)$ & $5.52 \pm 0.03(5.48-5.59)$ \\
\hline & FPH2015507 & $32.9 \pm 0.65(25.79-40.7)$ & $10.05 \pm 0.2(80.5-12.17)$ & $5.32 \pm 0.004(5.32-5.33)$ \\
\hline & FPH2015567 & $31.66 \pm 0.74(25.2-40.46)$ & $11.87 \pm 0.24(8.01-13.56)$ & $5.17 \pm 0.05(5.06-5.25)$ \\
\hline & FPH2015589 & $31.31 \pm 0.73(25.36-41.1)$ & $11.08 \pm 0.32(8.25-13.67)$ & $5.28 \pm 0.02(5.25-5.31)$ \\
\hline & FPH2015592 & $31.26 \pm 0.55(27.26-38.06)$ & $10.07 \pm 0.27(7.95-13.54)$ & $5.63 \pm 0.02(5.6-5.67)$ \\
\hline & FPH2015597 & $32.92 \pm 0.83(25.46-42.75)$ & $10.37 \pm 0.2(8.76-13.72)$ & $5.16 \pm 0.05(5.06-5.27)$ \\
\hline & FPH2016709 & $32.98 \pm 0.77(26.88-42.28)$ & $11.8 \pm 0.29(9.15-15.19)$ & $5.23 \pm 0.02(5.19-5.28)$ \\
\hline & FPH2016711 & $31.95 \pm 0.84(25.85-43.78)$ & $10.85 \pm 0.23(8.19-13.5)$ & $5.58 \pm 0.02(5.53-5.62)$ \\
\hline & Mean & $32.09 \pm 0.23 C^{x}$ & $10.77 \pm 0.09 \mathrm{~B}$ & $5.37 \pm 0.03 \mathrm{~A}$ \\
\hline \multirow[t]{2}{*}{ A. arborescens } & FPH2015395 & $29.05 \pm 00.67(22.47-35.37)$ & $11.84 \pm 0.21(9.21-15.11)$ & $5.26 \pm 0.05(5.2-5.37)$ \\
\hline & Mean & $29.05 \pm 00.67 \mathrm{D}$ & $11.84 \pm 0.21 \mathrm{~A}$ & $5.26 \pm 0.05 \mathrm{~A}$ \\
\hline \multirow[t]{3}{*}{ A. tenuissima } & FPH2015470 & $43.19 \pm 1.1(35.73-59.01)$ & $10.76 \pm 0.28(8.46-13.52)$ & $5.58 \pm 0.02(5.54-5.62)$ \\
\hline & FPH2015591 & $39.04 \pm 0.88(32.57-51.34)$ & $10.3 \pm 0.18(8.66-12.3)$ & $5.31 \pm 0.02(5.29-5.35)$ \\
\hline & Mean & $41.11 \pm 0.52 \mathrm{~A}$ & $10.53 \pm 0.2 \mathrm{~B}$ & $5.45 \pm 0.06 \mathrm{~A}$ \\
\hline \multirow[t]{2}{*}{ A. toxicogenica } & FPH2015338 & $37.29 \pm 0.66(30.38-44.79)$ & $10.7 \pm 0.23(8.81-13.88)$ & $5.31 \pm 0.01(5.29-5.32)$ \\
\hline & Mean & $37.29 \pm 0.66 \mathrm{~B}$ & $10.7 \pm 0.23 \mathrm{~B}$ & $5.31 \pm 0.01 \mathrm{~A}$ \\
\hline \multirow[t]{8}{*}{ Colletotrichum fioriniae } & FPH201314 & $10.57 \pm 0.21(8.9-13.24)$ & $3.71 \pm 0.11(2.94-5.72)$ & $3.02 \pm 0.03(2.97-3.08)$ \\
\hline & FPH201317 & $9.02 \pm 0.19(7.32-10.61)$ & $4.01 \pm 0.11(3.15-5.47)$ & $2.83 \pm 0.02(2.77-2.85)$ \\
\hline & FPH201319 & $9.86 \pm 0.13(8.25-11.93)$ & $4.39 \pm 0.07(3.51-5.12)$ & $2.51 \pm 0.08(2.39-2.69)$ \\
\hline & FPH2015392 & $6.13 \pm 0.17(4.37-8.41)$ & $3.74 \pm 0.08(3.06-5.17)$ & $2.69 \pm 0.11(2.52-2.96)$ \\
\hline & FPH2015466 & $11.17 \pm 0.14(9.77-12.74)$ & $4.81 \pm 0.09(4.03-5.77)$ & $2.99 \pm 0.01(2.97-3.01)$ \\
\hline & FPH2015564 & $11.17 \pm 0.13(9.88-12.5)$ & $4.81 \pm 0.09(4.03-5.75)$ & $3.02 \pm 0.02(2.97-3.06)$ \\
\hline & FPH2016720 & $10.45 \pm 0.24(8.01-12.38)$ & $4 \pm 0.11(2.94-5.52)$ & $3.25 \pm 0.1(3.02-3.43)$ \\
\hline & Mean & $9.77 \pm 0.13 \mathrm{~A}$ & $4.21 \pm 0.05 \mathrm{~B}$ & $2.9 \pm 0.06 \mathrm{~A}$ \\
\hline \multirow[t]{2}{*}{ C. nymphaeae } & FPH2015590 & $10.13 \pm 0.17(8.31-13.6)$ & $4.54 \pm 0.1(3.65-6.31)$ & $2.87 \pm 0.11(2.59-3.02)$ \\
\hline & Mean & $10.13 \pm 0.17 \mathrm{~A}$ & $4.54 \pm 0.1 \mathrm{~A}$ & $2.87 \pm 0.11 \mathrm{~A}$ \\
\hline \multirow[t]{6}{*}{ Diaporthe eres } & FPH201322 & $7.58 \pm 0.09(6.74-8.61)^{y}$ & $2.99 \pm 0.04(2.59-3.53)$ & $4.7 \pm 0.19(4.25-4.96)$ \\
\hline & & $24.73 \pm 0.52(18.91-29.65)^{\mathrm{z}}$ & $1.68 \pm 0.05(1.27-2.13)$ & \\
\hline & FPH2015394 & $6.38 \pm 0.12(5.04-8.01)$ & $2.89 \pm 0.06(2.21-3.82)$ & $5.59 \pm 0.12(5.31-5.8)$ \\
\hline & & $24.39 \pm 0.38(21.66-29.79)$ & $1.8 \pm 0.03(1.48-2.09)$ & \\
\hline & FPH2017229 & $6.87 \pm 0.08(6-7.58)$ & $2.59 \pm 0.05(2.15-3.32)$ & $4.89 \pm 0.09(4.76-5.05)$ \\
\hline & & $24.81 \pm 0.44(18.76-29.66)$ & $1.63 \pm 0.03(1.27-2.05)$ & \\
\hline \multirow[t]{2}{*}{ Epicoccum nigrum } & FPH2015417 & $20.27 \pm 0.27(17.37-23.26)$ & $18 \pm 0.3(14.12-22.18)$ & $3.43 \pm 0.02(3.38-3.45)$ \\
\hline & FPH2015505 & $19.85 \pm 0.31(17.05-23.78)$ & $17.23 \pm 0.32(13.17-21.71)$ & $3.57 \pm 0.05(3.48-3.69)$ \\
\hline
\end{tabular}

\footnotetext{
$\checkmark$ Values reported are mean \pm standard error (minimum-maximum size) of 30 randomly selected conidia.

w Values reported are mean \pm standard error (minimum-maximum mycelia growth rate).

${ }^{x}$ Values with the same letter in the same column within each fungal genus are not significantly different $(\alpha=0.05)$

y Alpha conidia.

z Beta conidia.
} 
orange to brown diffusible pigment in PDA. Dark pigmented conidia were produced in sporodochia, and were multicellular and globose to pyriform. These features were consistent with the description of Epicoccum nigrum Link (Mahadevakumar et al. 2014; Wu et al. 2017). Both ML and BI analyses using the ITS gene were in accordance with the results of morphological characterization as both isolates from this study clustered with E. nigrum reference strains (Chen et al. 2017; Valenzuela-Lopez et al. 2018; Fig. 7).
Pathogenicity tests. In experiment A, all isolates of A. alternata, C. fioriniae, D. ilicicola sp. nov., and E. nigrum were able to infect detached wounded mature holly fruit. Brown to black lesions developed from the inoculation point on all fruit inoculated with Alternaria and Colletotrichum isolates 2 DPI $(=100 \%$ incidence; data not shown). After 3 to 5 days, lesions enlarged to cover the whole fruit and sporulation occurred on the surface of the fruit (Fig. 8A and B). D. ilicicola sp. nov. caused a light salmon discoloration of the

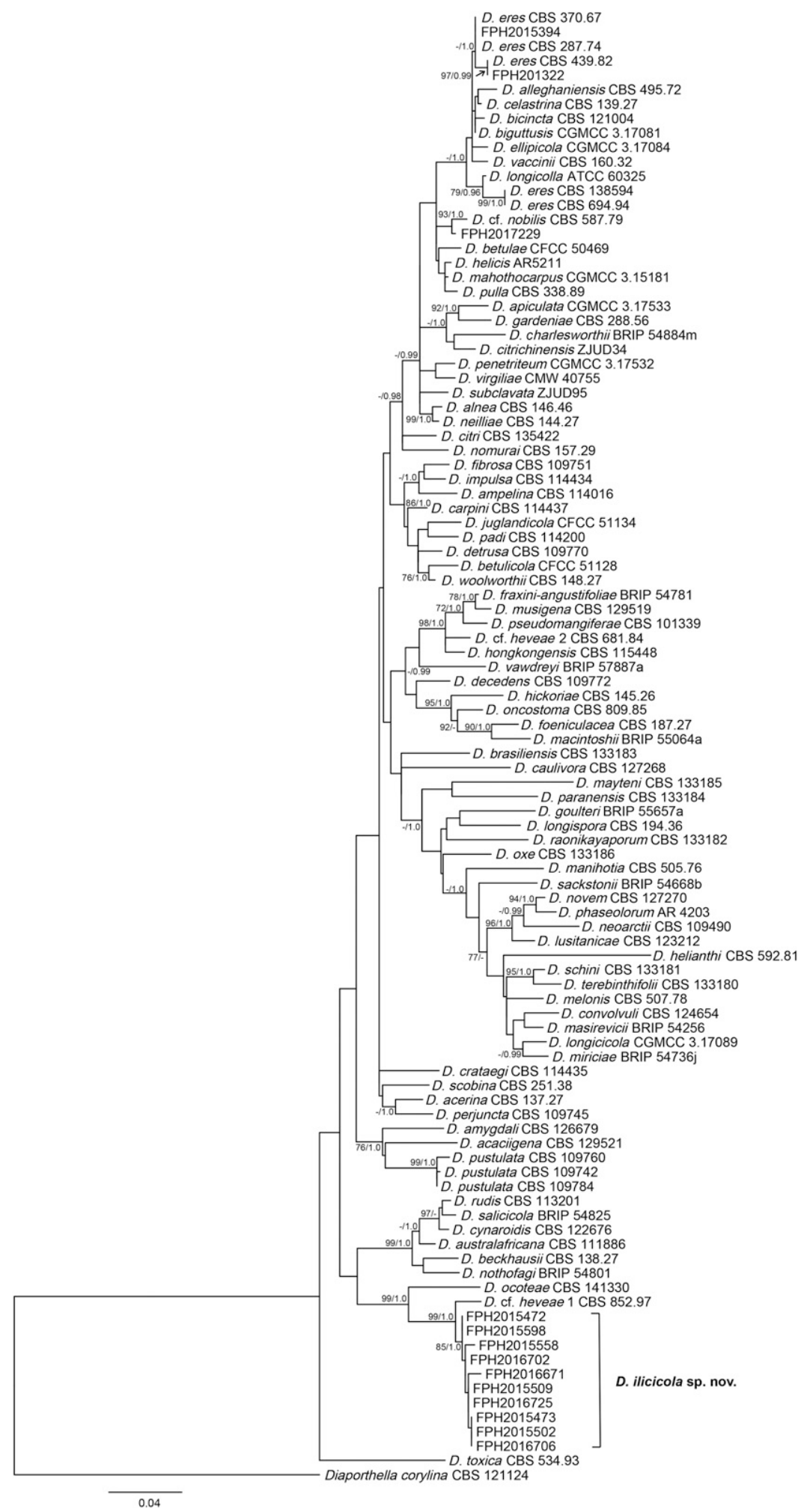

Fig. 4. Maximum likelihood (ML) analysis tree based on the General Time Reversible plus a gamma distribution model obtained from concatenated sequences of combined HIS, ITS, and TUB datasets of Diaporthe spp. The ML bootstrap support (BS) $\geq 70$ and Bayesian posterior probability (PP) $\geq 0.95$ values are indicated at the nodes (BS/PP). The Diaporthe ilicicola sp. nov. isolates from this study are highlighted. The tree was rooted to Diaporthella corylina (CBS 121124). 
fruit 1 week post-inoculation. Pycnidia exuding light yellow conidial masses (Fig. 8C) formed on the entire fruit 14 DPI (= $100 \%$ incidence; data not shown). Isolates of $D$. eres induced similar symptoms but after a longer period of time (3 to 4 weeks). Fruit rot incidence for D. eres isolates FPH201322, FPH2015394, and FPH2017229 was 45,70 , and $65 \%$, respectively (data not shown). Epicoccum isolates were able to induce similar symptoms to those caused by Alternaria and Colletotrichum; however, lesions remained limited to the point of inoculation (Fig. 8D) and disease incidence was relatively lower overall (60\%; data not shown).

In experiment $\mathrm{B}$, all four pathogens, regardless of the concentration of inoculum tested, were able to infect both wounded and unwounded detached fruit (data not shown). Disease incidence and severity on wounded fruit, however, were significantly higher than on unwounded fruit. On wounded fruit, disease incidence was above $88 \%$ for all pathogens 16 DPI (data not shown). Except for Epicoccum, disease severity increased along with inoculum concentration. Epicoccum inoculations resulted in low fruit rot severity, ranging from 5 to $11 \%$ at all concentrations (data not shown). On unwounded fruit, symptoms started to develop 24 DPI. No correlation pattern was observed between disease incidence and inoculum concentration. Disease incidence and severity for all treatments 42 DPI were 33 to $66 \%$ and 5 to $41 \%$, respectively. Among all the pathogeninoculum combinations tested, D. ilicicola sp. nov. at $10^{6}$ conidia $/ \mathrm{ml}$ caused the highest fruit rot incidence and severity.

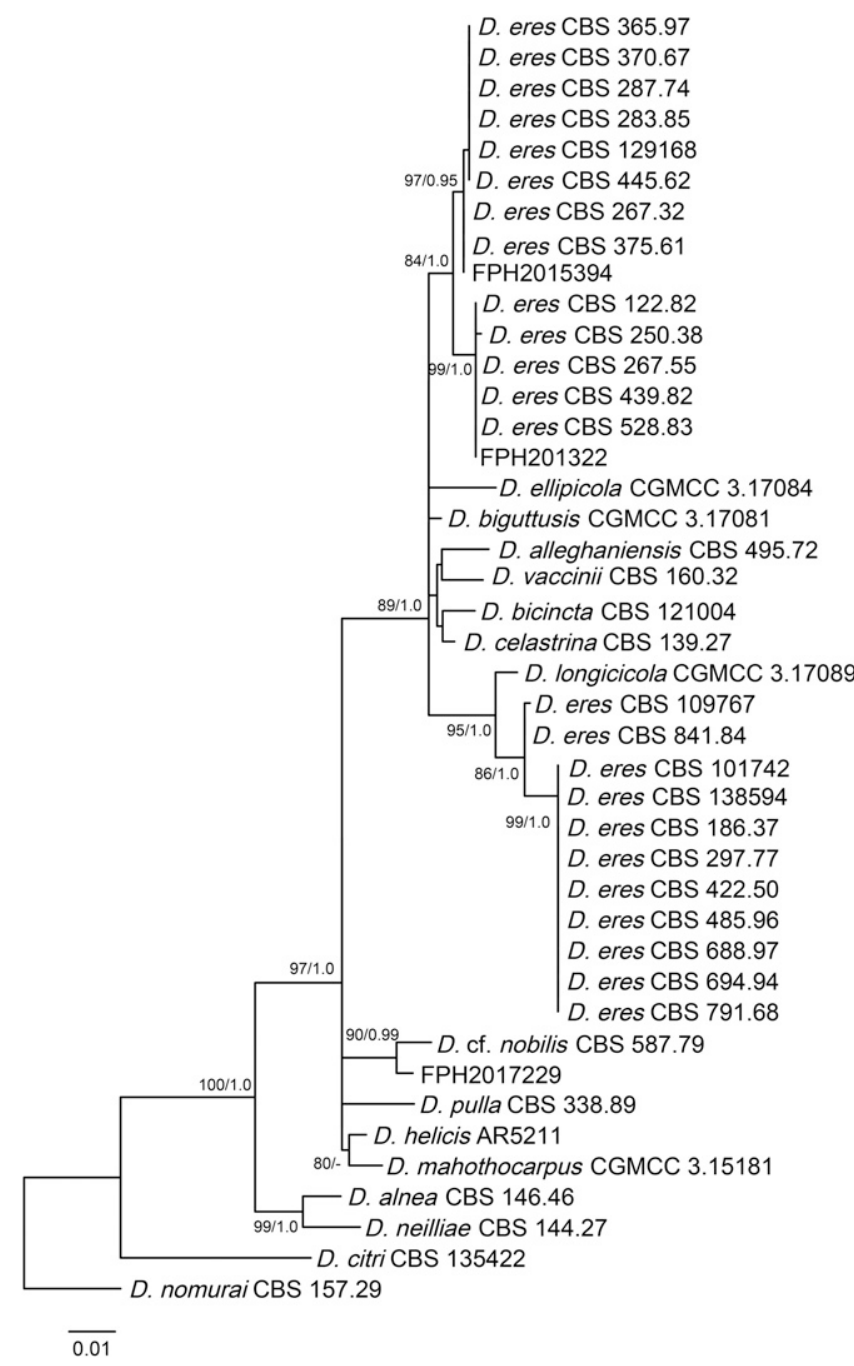

Fig. 5. Maximum likelihood (ML) phylogenetic tree based on the Hasegawa-KishinoYano plus a gamma distribution model obtained from combined HIS, ITS, and TUB datasets of Diaporthe spp. The ML bootstrap support (BS) $\geq 70$ and Bayesian posterior probability $(\mathrm{PP}) \geq 0.95$ values are indicated at the nodes (BS/PP). The tree was rooted with Diaporthe nomurai (CBS 157.29).
In experiment $\mathrm{C}$, all pathogen inoculum combinations at all concentrations were able to infect both wounded and unwounded fruit (Figs. 9 and 10). However, disease incidence and severity of combined pathogens on wounded and unwounded fruit were not significantly different from those infected by individual pathogens (Figs. 9 and 10). With the exception of E. nigrum, disease incidence and severity on wounded fruit for all the pathogen-concentration treatments were significantly higher than on unwounded fruit. Regardless of the presence/absence of wounding and the type of pathogen inoculum, fruit infected with $10^{5}$ conidia/ml resulted in significantly higher fruit rot incidence and severity compared with that inoculated with $10^{3}$ and $10^{2}$ conidia/ml. No rot symptoms developed on fruit in the control treatments in any of the three sets of experiments.

\section{Discussion}

Fruit rot is an emerging problem on deciduous holly that in recent years has been responsible for limiting crop production in ornamental nurseries across the Midwestern and Eastern U.S. Rot symptoms are characterized by black lesions on the surface of the fruit that eventually cause the whole fruit to soften and deteriorate. This study represents the first attempt to elucidate the etiology of this disease through characterization of the fungi associated with symptomatic tissues.

Seven different fungal species including A. alternata, A. arborescens, $C$. fioriniae, $C$. nymphaeae, $D$. ilicicola sp. nov., $D$. eres, and E. nigrum that were commonly associated with symptomatic deciduous holly samples were studied using morphological, phylogenetic, and pathogenicity characterizations. Among these, A. alternata and D. ilicicola sp. nov. were predominant. Overall, Colletotrichum and Epicoccum species were only occasionally isolated from fruit lesions, indicating that their role in the disease complex may be limited. Findings were supported by extensive sampling over multiple years and locations, including commercial nurseries, landscapes, and arboreta, mainly in Ohio but also in a few other eastern U.S. states.

The different fungal species recovered from the symptomatic samples did not seem to be associated with a specific sampling location. Instead, multiple species were found within the same planting at all locations except one arboretum in North Carolina, where only D. ilicicola sp. nov. was isolated. In particular, A. alternata and D. ilicicola sp. nov. were found within the same planting from more than half of the sampling locations across Ohio, Pennsylvania, and Massachusetts (data not shown). These findings contribute to supporting

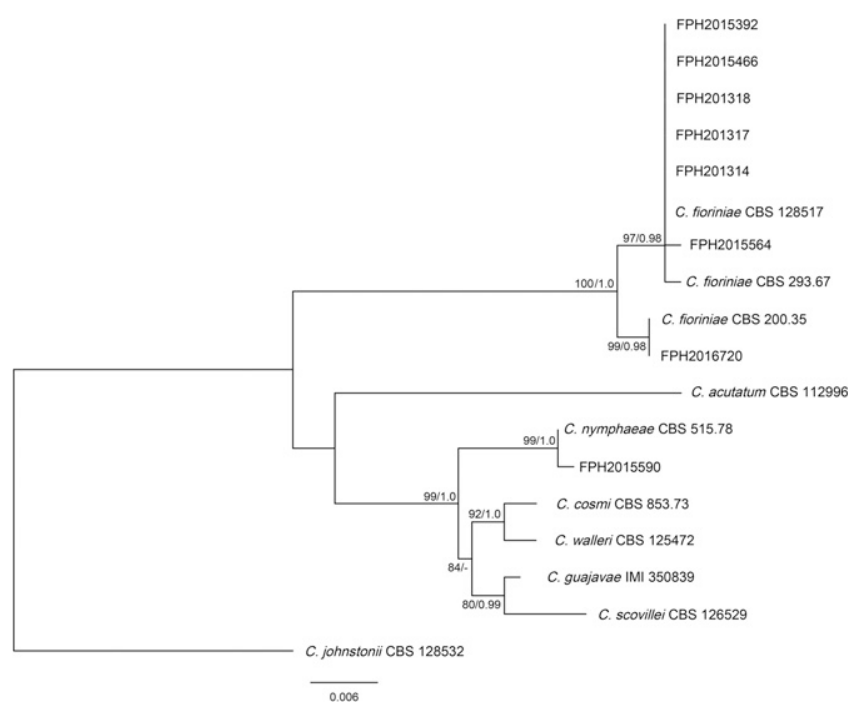

Fig. 6. Maximum likelihood (ML) phylogenetic tree based on the Hasegawa-KishinoYano plus a gamma distribution model obtained from combined GADPH and TUB datasets of Colletotrichum spp. The tree with the highest log likelihood $(-1,486.05)$ is shown. The ML bootstrap support (BS) $\geq 70$ and Bayesian posterior probability (PP) $\geq 0.95$ values are indicated at the nodes (BS/PP). The tree was rooted with Colletotrichum johnstonii (CBS 128532). 
our conclusion that $A$. alternata and $D$. ilicicola sp. nov. are the primary pathogens associated with fruit rot of deciduous holly.

Symptoms caused by the individual pathogens were not distinct. Additionally, fungi were not always recovered from rotten fruit, which indicates that some of the symptoms may have been due to other causes, possibly including bacterial infection, environmental stresses, physiological disorders, or mechanical injury. It has been observed that discoloration or blackening of deciduous holly fruit may occur due to cold weather or frost (Galle 1997). In our study, we consistently observed that early defoliation was associated with fruit rot in the field. Loss of leaves could expose fruit directly to the sunlight and possibly result in sunscald (Barber and Sharpe 1971). In this study, however, sunscald on fruit was not observed. Olatinwo et al. (2003) also found no fungi associated with some rotten fruit in the cranberry fruit rot disease complex, and speculated that it could be due to physiological breakdown or mechanical injury. While the focus of this study was to characterize the fungal pathogens associated with fruit rot of deciduous holly, the roles of other factors causing fruit rot should be further investigated.

Morphological characteristics were used to initially assign the isolated fungi to different genera. Within each genus, detailed morphological comparisons allowed us to separate isolates into several groups. Alternaria isolates in this study were identified as smallspored and grouped into four morphological groups: A. alternata, A. arborescens, A. tenuissima, and A. toxicogenica mainly based on conidial morphology and sporulation patterns (Simmons 2007). However, it was impossible to rely solely on morphological characistics for species identification because of the extensive number of species, overlapping morphological characteristics, and variable growth conditions. Several studies emphasize the importance of using combined morphological and molecular characterization for accurate identification of Alternaria species (Andrew et al. 2009; Armitage et al. 2015; Harteveld et al. 2013; Lawrence et al. 2013). In this study, we selected the endopolygalacturonase gene (endoPG) and two anonymous genomic regions OPA1-3 and OPA10-2 that were successfully used in previous studies to distinguish small-spored Alternaria species (Andrew et al. 2009; Peever et al. 2004; Woudenberg et al. 2015) and we used concatenated sequences of these three loci to carry out phylogenetic analyses. Isolates morphologically identified as A. arborescens and $A$. toxicogenica clustered with reference strains of these same species included in the analysis. However, isolates morphologically identified as A. alternata and A. tenuissima distributed throughout the phylogenetic tree and were not grouped into any specific clade. These results were in agreement with other studies that used multilocus phylogenetic analyses in which A. alternata and A. tenuissima could not be separated (Andrew et al. 2009; da Cruz Cabral et al. 2017; Luo et al. 2017; Woudenberg et al. 2015). Therefore, Andrew et al. (2009) proposed the separation of $A$. alternata and $A$. arborescens, but combined other morphospecies including $A$. alternata, A. tenuissima, and A. toxicogenica into one single species, namely A. alternata. A recent taxonomic study by Woudenberg et al. (2015) using multilocus phylogeny supported the aforementioned study and suggested merging 35 morphospecies of Alternaria into A. alternata. Accordingly, we concluded that species of Alternaria associated with fruit rot of deciduous holly included A. alternata and A. arborescens.

The use of a combination of morphological and multilocus phylogenetic analyses is strongly suggested in the literature to delimit species boundaries of Diaporthe (Gomes et al. 2013; Santos et al. 2017; Udayanga et al. 2012, 2014a). In our study, morphological characterization and phylogenetic studies of concatenated sequences of three loci (ITS+HIS+TUB) were used to identify Diaporthe spp. associated with fruit rot of deciduous holly. Several species of Diaporthe have been previously reported from dead twigs or leaves of Ilex spp., including D. eres, D. hongkongensis R.R. Gomes, C. Glienke \& Crous, D. ilicis (Ellis \& Everh.) Wehm., D. oncostoma (Duby) Fuckel, D. oxyspora (Peck) Sacc., D. pardalota (Mont.) Nitschke ex Fuckel, and D. rudis (Fr.) Nitschke (Cash 1952; Gao et al. 2016, 2017; Gomes et al. 2013; Guba and Stevenson 1963;
Wehmeyer 1933). However, only a few sequences of reference strains of these species from Ilex are available in the literature (i.e., D. eres [CBS 370.67 and CBS 694.94]; D. oncostoma [CBS 809.85]; Gomes et al. 2013). For D. rudis and D. hongkongensis, sequences of isolates from Ilex were not available; therefore the ex-type and ex-epitype of these two species (CBS 113201 and CBS 115448) were included in the analyses (Gomes et al. 2013). D. ilicis, D. oxyspora, and D. pardalota were originally reported from Ilex in 1933. Sequences of these three species, regardless of the host on which they were found, were also not available and were not included in the phylogenetic studies. As a result of multilocus ML and BI analyses, 10 of 13 isolates included in the present study did not cluster with any of the above species, but grouped together in an unnamed monophyletic clade closely related to a reference strain of $D$. cf. heveae 1 (CBS 852.97) isolated from Heveae brasiliensis in Brazil. Sequences from a total of six D. cf. heveae isolates, comprising unpublished sources, were initially included in the phylogenetic analysis and subsequently removed to include published sources only. It is to be noted that even in the initial analysis all the isolates from our study grouped in a different clade. The two $D$. cf. heveae reference isolates that were left (CBS 852.97 and CBS 681.84), however, were reported to be sterile (Gomes et al. 2013), so morphological comparisons between isolates was not feasible. Additionally, morphological characterizations of our 10 isolates differed from the original descriptions of $D$. ilicis, D. oxyspora, and D. pardalota available in the literature (Wehmeyer 1933). At the time this manuscript is being prepared, no new record of morphological or molecular characterization of these three species has been found. Additionally, these species had not been found to infect fruit of Ilex, either. Based on detailed morphological characterization and molecular analysis of our isolates, we hereby propose a new species description, namely $D$. ilicicola sp. nov. The genus Diaporthe consists of species that range from host specific to those with a wide host range. In this study, only Ilex fruit was inoculated to test pathogenicity of our D. ilicicola sp. nov. isolates. Cross-inoculation of D. ilicicola sp. nov. on other fruit crops may provide valuable information for further species characterization.

The remaining three Diaporthe isolates included in our study were identified as belonging to the $D$. eres species complex based on both morphological and molecular analyses. To better understand

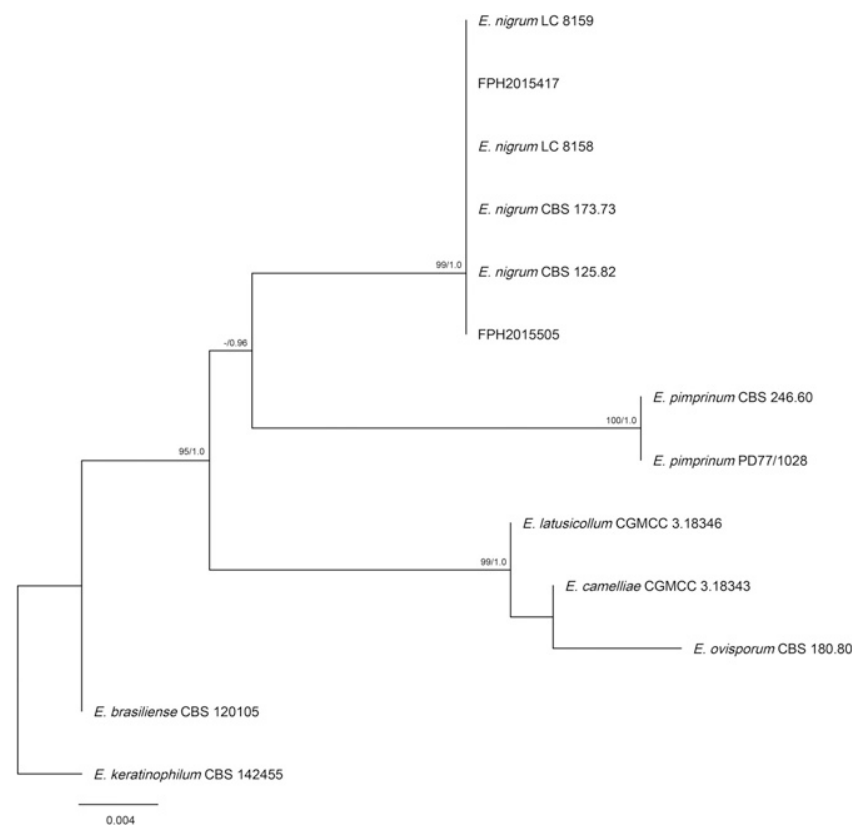

Fig. 7. Phylogenetic tree from maximum likelihood (ML) analysis based on the Hasegawa-Kishino-Yano plus a gamma distribution model obtained from ITS datasets of Epicoccum spp. The ML bootstrap support (BS) $\geq 70$ and Bayesian posterior probability (PP) $\geq 0.95$ values are indicated at the nodes (BS/PP). The tree was rooted with Epicoccum keratinophilum (CBS 142455). 
the relationship of these three isolates within the $D$. eres species complex, a second phylogenetic analysis was conducted using additional reference strains that were within or closely related to the $D$. eres species complex. According to the new analysis, two of the three isolates (FPH201322 and FPH2015394) clustered with D. eres reference strains, including one recovered from Ilex (CBS 370.67), while one isolate (FPH2017229) grouped with D. cf. nobilis (CBS 587.79). It is to be noted that alpha and beta conidia of FPH2017229 were significantly narrower compared with FPH201322 and FPH2015394, possibly indicating species differences. Our findings are in agreement with previous phylogenetic studies where separate clades for $D$. eres and $D$. nobilis were obtained (Baumgartner et al. 2013; Gomes et al. 2013; Lawrence et al. 2015), but are in disagreement with a study that specifically addressed the D. eres species complex (Udayanga et al. 2014a), in which D. nobilis was not recognized as a separate species. It is to be noted that in the latter case, ITS was not included in the multilocus phylogenetic analysis as this gene was considered as having too much variability within the genus and was discordant to other trees generated in the study. While the analysis of additional loci may be needed to further differentiate our three isolates within the $D$. eres species complex, due to the relatively low frequency
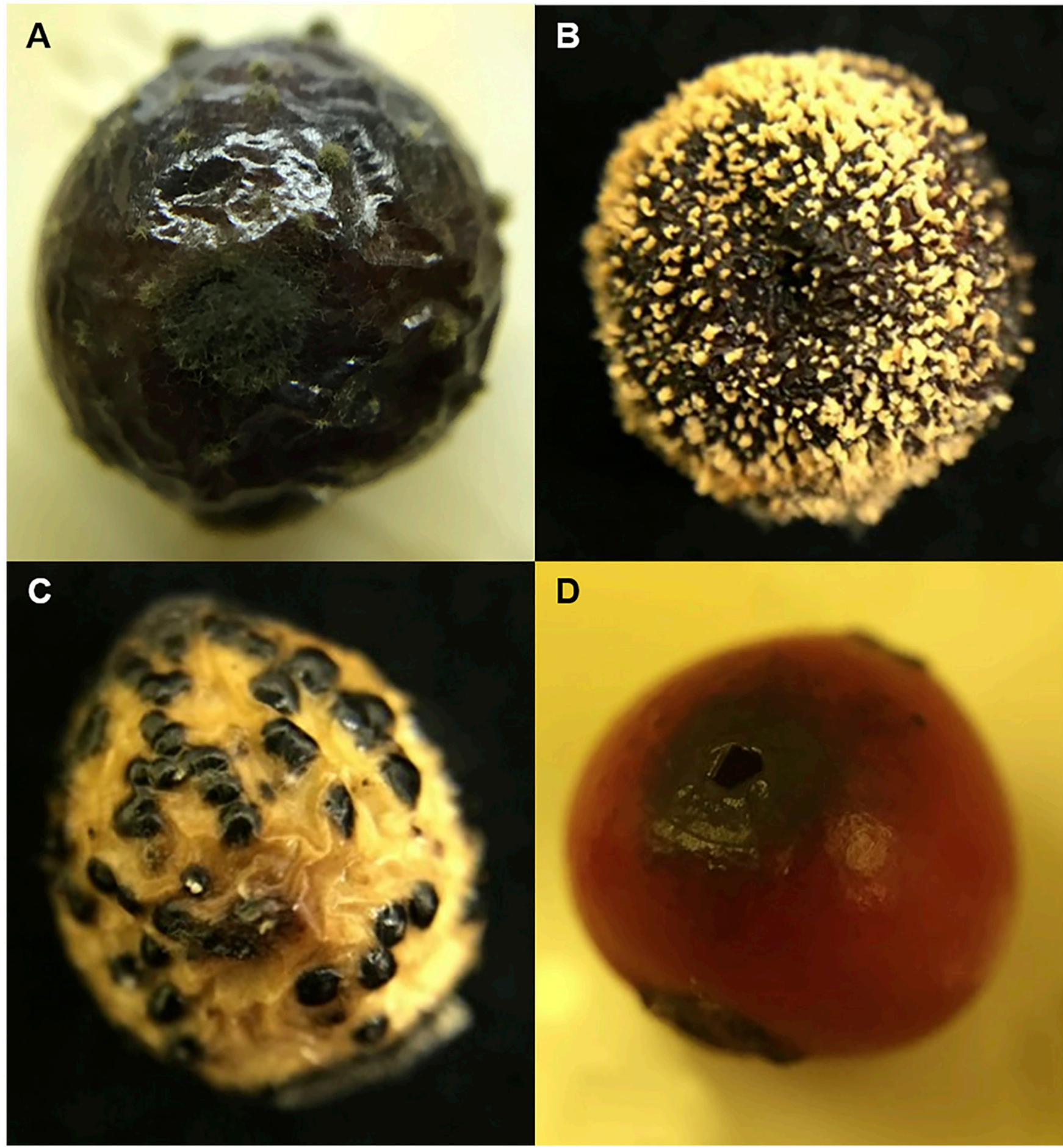

D

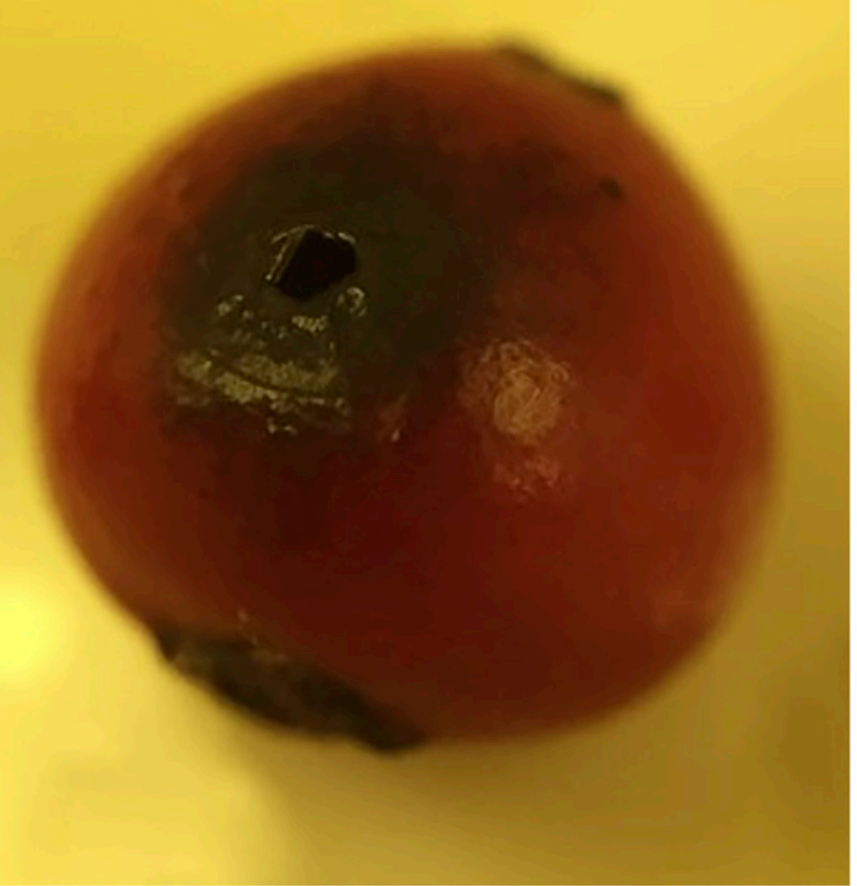

Fig. 8. Symptoms and signs on detached holly fruit used in the pathogenicity experiments 10 days postinoculation. A, Alternaria alternata; B, Colletotrichum fioriniae; C, Diaporthe ilicicola sp. nov.; D, Epicoccum nigrum). 
of isolation of these fungi in our study, we did not pursue additional identification. Both $D$. eres and D. nobilis are reported as minor pathogens causing blight, cankers, leaf spots, and fruit rot on a broad range of host families of woody plants worldwide (Baumgartner et al. 2013; Choi et al. 2017; Farr and Rossman 2018; Kaliterna et al. 2012; Lawrence et al. 2015; Li et al. 2017; Lorenzini and Zapparoli 2018; Thomidis and Michailides 2009; Zhang et al. 2016). Indeed, in our pathogenicity experiments, $D$. eres isolates caused relatively lower fruit rot incidence compared with D. ilicicola sp. nov. and it took a longer period of time for the inoculated fruit to develop symptoms. All D. ilicicola sp. nov. isolates tested resulted in $100 \%$ disease incidence with symptoms visible in less than a week, compared with 45 to $70 \%$ incidence seen for $D$. eres that took 21 to 28 days to become evident. Based on these observations, fungi in the $D$. eres species complex should be considered minor pathogens causing fruit rot of deciduous holly.

Selected isolates belonging to the two predominantly isolated pathogen species (A. alternata and D. ilicicola sp. nov.), along with possibly minor pathogens such as $C$. fioriniae and $E$. nigrum, were included in three sets of pathogenicity experiments that not only tested their ability to cause disease but also aimed to understand the role of wounding and inoculum concentration on disease

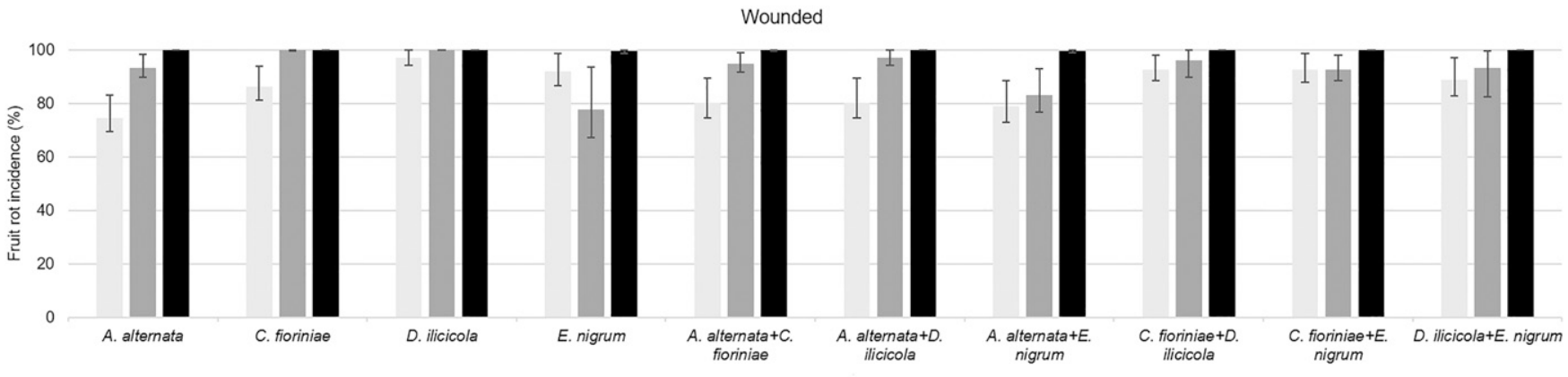

Unwounded

$$
100
$$

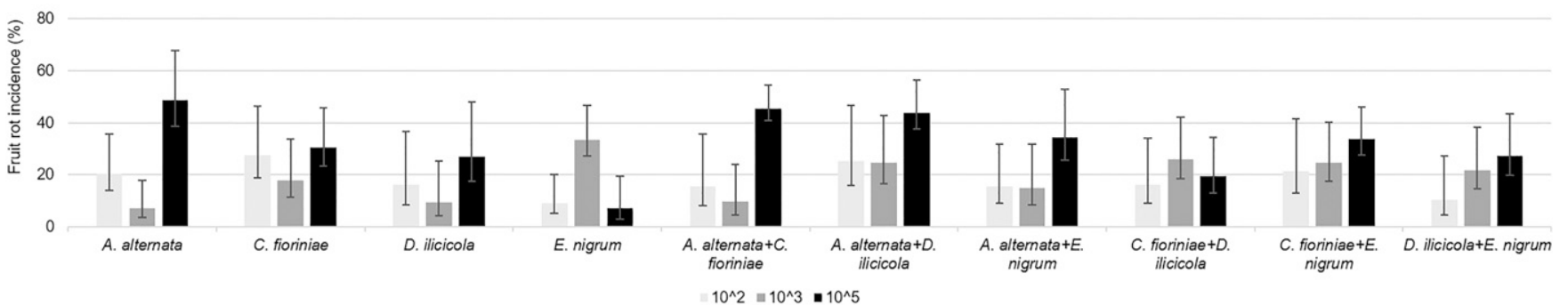

Fig. 9. Fruit rot incidence recorded on wounded and unwounded fruit inoculated in pathogenicity experiment $\mathrm{C}$ with individual and combined pathogens at all inoculum concentrations. Bars indicate $95 \%$ confidence intervals.
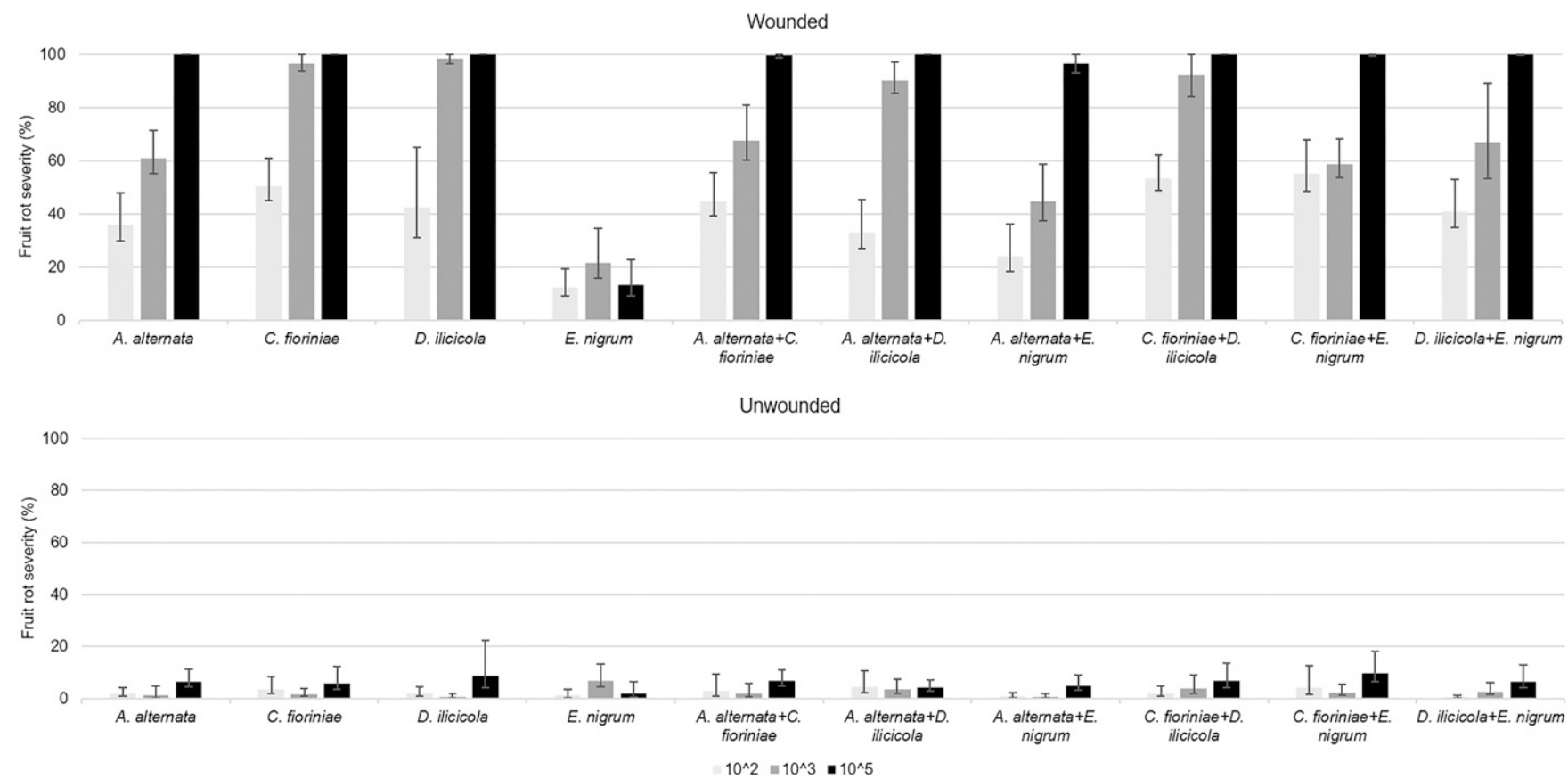

Fig. 10. Fruit rot severity recorded on wounded and unwounded fruit inoculated in pathogenicity experiment $\mathrm{C}$ with individual and combined pathogens at all inoculum concentrations. Bars indicate 95\% confidence intervals. 
development. All isolates were able to infect detached mature holly fruit and induce rot symptoms, and all were reisolated from the symptomatic tissues, which fulfilled Koch's postulates. Disease incidence and severity levels, however, were overall much higher in fruit that had been wounded compared with unwounded fruit, indicating that fruit injury, either biotic or abiotic in nature, could result in higher disease levels in the field. Higher inoculum concentration always resulted in more severe disease, suggesting that practices that can decrease inoculum concentration (e.g., removal of infected plant material, pruning, or chemical applications) could lower disease levels in the field. Since occasionally multiple pathogens were isolated from the same lesion on the original field samples, individual inoculum and combination of pathogens were also tested. Similar disease incidence and severity resulted from single versus combined pathogen inoculations. It is to be acknowledged that all inoculation experiments in this study were conducted in the laboratory where fruit was maintained in a controlled environment at optimal temperature and humidity levels, which may have favored pathogen activity. However, a separate set of inoculations whose results are partially reported elsewhere (Lin et al. 2017) were made in the winter of 2016 on wounded and unwounded fruit of deciduous holly cultivar Sparkleberry maintained outdoors in a container trial. In that experiment, fruit was inoculated with some of the same isolates used in the detached fruit assays reported in this study, and naturally exposed to fluctuating environmental conditions. While disease incidence was overall lower in the outdoor trial, all four pathogens (A. alternata, C. fioriniae, D. ilicicola sp. nov., and $E$. nigrum) successfully infected wounded mature fruit, caused the same symptoms observed on the detached fruit assays, and were reisolated from the lesions, which fulfilled Koch's postulates. However, no infection was observed on unwounded fruit in planta. Further studies investigating the possible pathways of fruit infection in natural settings are underway and will complement these preliminary observations.

This study identified and characterized various fungal pathogens associated with fruit rot of deciduous holly, which represents a first step in the understanding of this disease complex. Further research is being conducted to investigate the sources of pathogen inoculum in the field, to understand when pathogens infect the plant during the growing season, and to identify the environmental factors that favor disease development. Precise information about the disease cycle and disease epidemiology is needed to ultimately provide nursery growers with effective management recommendations.

\section{Acknowledgments}

The research described in this paper represents a portion of the dissertation submitted by S. Lin to the Office of Graduate Studies of The Ohio State University to partially fulfill requirements for the Ph.D. degree in Plant Pathology. The authors thank all the winterberry growers in Ohio, Pennsylvania, and Massachusetts who provided many of the fruit samples used in this study. The authors also thank Dr. F. P. Trouillas for assistance in the description of the new Diaporthe species, as well as Dr. J. R. Urbez-Torres and Dr. G. Marchi for critical review of this manuscript prior to submission.

\section{Literature Cited}

Andrew, M., Peever, T. L., and Pryor, B. M. 2009. An expanded multilocus phylogeny does not resolve morphological species within the small-spored Alternaria species complex. Mycologia 101:95-109.

Armitage, A. D., Barbara, D. J., Harrison, R. J., Lane, C. R., Sreenivasaprasad, S., Woodhall, J. W., and Clarkson, J. P. 2015. Discrete lineages within Alternaria alternata species group: Identification using new highly variable loci and support from morphological characters. Fungal Biol. 119:994-1006.

Barber, H. N., and Sharpe, P. J. H. 1971. Genetics and physiology of sunscald of fruits. Agric. Meteorol. 8:175-191.

Barnett, H. L., and Hunter, B. B. 1998. Illustrated genera of imperfect fungi, 4th Ed. American Phytopathological Society, St. Paul, MN.

Baumgartner, K., Fujiyoshi, P. T., Travadon, R., Castlebury, L. A., Wilcox, W. F., and Rolshausen, P. E. 2013. Characterization of species of Diaporthe from wood cankers of grape in Eastern North American vineyards. Plant Dis. 97: 912-920.

Biggs, A. R. 2004. Effect of inoculum concentration and calcium salts on infection of apple fruit by Botryosphaeria dothidea. Plant Dis. 88:147-151.

Cash, E. K. 1952. A record of the fungi named by J.B. Ellis (Part 1). U.S.D.A. Spec. Publ. 2:1-165.
Chen, Q., Hou, L. W., Duan, W. J., Crous, P. W., and Cai, L. 2017. Didymellaceae revisited. Stud. Mycol. 87:105-159.

Choi, I. Y., Joa, J. H., Cho, S. W., Lee, W. H., Galea, V., and Shin, H. D. 2017. Occurrence of stem and shoot cankers caused by Phomopsis fukushii on mango. Australas. Plant Dis. Notes 12:56.

Crous, P. W., Groenewald, J. Z., Risede, J. M., and Hywel-Jones, N. L. 2004 Calonectria species and their Cylindrocladium anamorphs: species with sphaeropedunculate vesicles. Stud. Mycol. 50:415-430.

Curry, K. J., Abril, M., Avant, J. B., and Smith, B. J. 2002. Strawberry anthracnose: histopathology of Colletotrichum acutatum and C. fragariae. Phytopathology 92:1055-1063.

da Cruz Cabral, L., Rodriguero, M., Stenglein, S., Nielsen, K. F., and Patriarca, A 2017. Characterization of small-spored Alternaria from Argentinean crops through a polyphasic approach. Int. J. Food Microbiol. 257:206-215.

Damm, U., Cannon, P. F., Woudenberg, J. H., and Crous, P. W. 2012. The Colletotrichum acutatum species complex. Stud. Mycol. 73:37-113.

Dissanayake, A. J., Liu, M., Zhang, W., Chen, Z., Udayanga, D., Chukeatirote, E., Li, X. H., Yan, J. Y., and Hyde, K. D. 2015. Morphological and molecular characterization of Diaporthe species associated with grapevine trunk disease in China. Fungal Biol. 119:283-294.

Dissanayake, A. J., Phillips, A. J. L., Hyde, K. D., Yan, J. Y., and Li, X. H. 2017 The current status of species in Diaporthe. Mycosphere. 8:1106-1156.

Du, Z., Fan, X. L., Hyde, K. D., Yang, Q., Liang, Y. M., and Tian, C. M. 2016. Phylogeny and morphology reveal two new species of Diaporthe from Betula spp. in China. Phytotaxa 269:90-102.

Farr, D. F., and Rossman, A. Y. 2018. Fungal Databases, U.S. National Fungus Collections, ARS, USDA. Retrieved from https://nt.ars-grin.gov/ fungaldatabases/.

Fernald, M. L. 1950. Gray's Manual of Botany. American Book Company, New York.

Galle, F. C. 1997. Hollies: The Genus Ilex. Timber Press, Portland, Oregon.

Gao, L. L., Zhang, Q., Sun, X. Y., Jiang, L., Zhang, R., Sun, G. Y., Zha, Y. L., and Biggs, A. R. 2013. Etiology of moldy core, core browning, and core rot of Fuji apple in China. Plant Dis. 97:510-516.

Gao, Y., Liu, F., and Cai, L. 2016. Unravelling Diaporthe species associated with Camellia. Syst. Biodivers. 14:102-117.

Gao, Y., Liu, F., Duan, W. J., Crous, P. W., and Cai, L. 2017. Diaporthe is paraphyletic. IMA Fungus 8:153-187.

Glass, N. L., and Donaldson, G. 1995. Development of primer sets designed for use with PCR to amplify conserved genes from filamentous Ascomycetes. Appl Environ. Microbiol. 61:1323-1330.

Gomes, R. R., Glienke, C., Videira, S. I. R., Lombard, L., Groenewald, J. Z., and Crous, P. W. 2013. Diaporthe: A genus of endophytic, saprobic and plant pathogenic fungi. Persoonia 31:1-41.

Guba, E. F., and Stevenson, J. A. 1963. Fungus and nematode inhabitants and diseases of holly (Ilex). Mass Agric. Exp. Sta. Bull. 530:1-43.

Harteveld, D. O. C., Akinsanmi, O. A., and Drenth, A. 2013. Multiple Alternaria species groups are associated with leaf blotch and fruit spot diseases of apple in Australia. Plant Pathol. 62:289-297.

Howard, C. M., Maas, J. L., Chandler, C. K., and Albregts, E. E. 1992 Anthracnose of strawberry caused by the Colletotrichum complex in Florida. Plant Dis. 76:976-981.

Huang, F., Hou, X., Dewdney, M. M., Fu, Y., Chen, G., Hyde, K. D., and Li, H. 2013. Diaporthe species occurring on citrus in China. Fungal Divers. 61: 237-250.

Huang, F., Udayanga, D., Wang, X., Hou, X., Mei, X., Fu, Y., Hyde, K. D., and Li, H. 2015. Endophytic Diaporthe associated with Citrus: A phylogenetic reassessment with seven new species from China. Fungal Biol. 119:331-347.

Kaliterna, J., Miličević, T., and Cvjetković, B. 2012. Grapevine trunk diseases associated with fungi from the Diaporthaceae family in Croatian vineyards. Arch Ind Hyg Toxicol 63:471-478.

Kimura, M. 1980. A simple method for estimating evolutionary rate of base substitutions through comparative studies of nucleotide sequences. J. Mol. Evol. 16:111-120.

Kumar, S., Stecher, G., and Tamura, K. 2016. MEGA7: Molecular Evolutionary Genetics Analysis Version 7.0 for Bigger Datasets. Mol. Biol. Evol. 33:1870-1874.

Lawrence, D. P., Gannibal, P. B., Peever, T. L., and Pryor, B. M. 2013. The sections of Alternaria: formalizing species-group concepts. Mycologia 105:530-546.

Lawrence, D. P., Travadon, R., and Baumgartner, K. 2015. Diversity of Diaporthe species associated with wood cankers of fruit and nut crops in northern California. Mycologia 107:926-940.

Li, Y., Tan, P., and Zhao, D. G. 2017. Diaporthe nobilis, a new record on Camellia sinensis in Guizhou Province, China. Mycosphere 8:1-8.

Lin, S., Taylor, N. J., and Peduto Hand, F. 2017. Determining the timing of host susceptibility to infection by fungal pathogens associated with fruit rot disease of winterberry holly. Phytopathology 107:S5.34.

Lorenzini, M., and Zapparoli, G. 2018. Identification of Pestalotiopsis bicilita, Diplodia seriata and Diaporthe eres causing fruit rot in withered grapes in Italy. Eur. J. Plant Pathol. 151:1089-1093.

Luo, Y., Hou, L., Förster, H., Pryor, B., and Adaskaveg, J. E. 2017. Identification of Alternaria species causing heart rot of pomegranates in California. Plant Dis. 101:421-427. 
Machingambi, N. M., Dreyer, L. L., Oberlander, K. C., Roux, J., and Roets, F. 2015. Death of endemic Virgilia oroboides trees in South Africa caused by Diaporthe virgiliae sp. nov. Plant Pathol. 64:1149-1156.

Mahadevakumar, S., Jayaramaiah, K. M., and Janardhana, G. R. 2014. First report of leaf spot disease caused by Epicoccum nigrum on Lablab purpureus in India. Plant Dis. 98:284.

McKay, S. F., Freeman, S., Minz, D., Maymon, M., Sedgley, M., Collins, G. C., and Scott, E. S. 2009. Morphological, genetic, and pathogenic characterization of Colletotrichum acutatum, the cause of anthracnose of almond in Australia. Phytopathology 99:985-995.

Milholland, R. D., and Daykin, M. E. 1983. Blueberry fruit rot caused by Phomopsis vaccinii. Plant Dis. 67:325-326.

Mishra, B., and Thines, M. 2014. siMBa—a simple graphical user interface for the Bayesian phylogenetic inference program MrBayes. Mycol. Prog. 13:1010.

Mostert, L., Alan, J. L. P., Crous, P. W., and Kang, J. C. 2001. Species of Phomopsis and a Libertella sp. occurring on grapevines with specific reference to South Africa: morphological, cultural, molecular and pathological characterization. Mycologia 93:146-167.

Munir, M., Amsden, B., Dixon, E., Vaillancourt, L., and Gauthier, N. A. W. 2016. Characterization of Colletotrichum species causing bitter rot of apple in Kentucky orchards. Plant Dis. 100:2194-2203.

NASS. 2014. Cut flowers, Ilex - sales, measured in \$. https://quickstats.nass.usda. gov/results/506C6C00-A626-314B-AE8B-74588F150F45.

O'Donnell, K., and Cigelnik, E. 1997. Two divergent intragenomic rDNA ITS2 types within a monophyletic lineage of the fungus Fusarium are nonorthologous. Mol. Phylogenet. Evol. 7:103-116.

Olatinwo, R. O., Hanson, E. J., and Schilder, A. M. C. 2003. A first assessment of the cranberry fruit rot complex in Michigan. Plant Dis. 87:550-556.

Peever, T. L., Su, G., Carpenter-Boggs, L., and Timmer, L. W. 2004. Molecular systematics of citrus-associated Alternaria species. Mycologia 96:119-134.

Rambaut, A. 2016. FigTree tree figure drawing tool version 1.4.3. Institute of Evolutionary Biology, University of Edinburgh.

Ronquist, F., and Huelsenbeck, J. P. 2003. MRBAYES 3: Bayesian phylogenetic inference under mixed models. Bioinformatics 19:1572-1574.

Rotondo, F., Collina, M., Brunelli, A., and Pryor, B. M. 2012. Comparison of Alternaria spp. collected in Italy from apple with A. mali and other AM-toxin producing strains. Phytopathology 102:1130-1142.

Santos, L., Alves, A., and Alves, R. 2017. Evaluating multi-locus phylogenies for species boundaries determination in the genus Diaporthe. PeerJ 5:e3120.

Shivas, R. G., and Tan, Y. P. 2009. A taxonomic re-assessment of Colletotrichum acutatum, intro-ducing $C$. fioriniae comb. et stat. nov. and $C$. simmondsii sp. nov. Fungal Divers. 39:111-122.

Simmons, E. G. 2007. Alternaria: An identification manual. CBS Fungal Biodiversity Centre, Utrecht, The Netherlands.

Tan, Y. P., Edwards, J., Grice, K. R. E., and Shivas, R. G. 2013. Molecular phylogenetic analysis reveals six new species of Diaporthe from Australia. Fungal Divers. 61:251-260.

Templeton, M. D., Rikkerink, E. H. A., Solon, S. L., and Crowhurst, R. N. 1992. Cloning and molecular characterization of the glyceraldehyde-3-phosphate dehydrogenase encoding gene and cDNA from the plant pathogenic fungus Glomerella cingulata. Gene 122:225-230.

Thomidis, T., and Michailides, T. J. 2009. Studies on Diaporthe eres as a new pathogen of peach trees in Greece. Plant Dis. 93:1293-1297.

Thompson, S. M., Neate, S. M., Thompson, S. M., Aitken, E. A. B., Tan, Y. P., Shivas, R. G., Morin, L., and Bissett, A. 2015. Green and brown bridges between weeds and crops reveal novel Diaporthe species in Australia. Persoonia - Mol. Phylogeny Evol. Fungi 35:39-49.

Udayanga, D., Castlebury, L. A., Rossman, A. Y., Chukeatirote, E., and Hyde, K. D. 2014a. Insights into the genus Diaporthe: phylogenetic species delimitation in the D. eres species complex. Fungal Divers. 67:203-229.

Udayanga, D., Castlebury, L. A., Rossman, A. Y., Chukeatirote, E., and Hyde, K. D. 2015. The Diaporthe sojae species complex: phylogenetic reassessment of pathogens associated with soybean, cucurbits and other field crops. Fungal Biol. 119:383-407.

Udayanga, D., Castlebury, L. A., Rossman, A. Y., and Hyde, K. D. 2014b. Species limits in Diaporthe: molecular re-assessment of D. citri, D. cytosporella, D. foeniculina and D. rudis. Persoonia - Mol. Phylogeny Evol. Fungi 32:83-101.

Udayanga, D., Liu, X., Crous, P. W., McKenzie, E. H. C., Chukeatirote, E., and Hyde, K. D. 2012. A multi-locus phylogenetic evaluation of Diaporthe (Phomopsis). Fungal Divers. 56:157-171.

Valenzuela-Lopez, N., Cano-Lira, J. F., Guarro, J., Sutton, D. A., Wiederhold, N., Crous, P. W., and Stchigel, A. M. 2018. Coelomycetous Dothideomycetes with emphasis on the families Cucurbitariaceae and Didymellaceae. Stud. Mycol. 90:1-69.

Velho, A., Alaniz, S., Casanova, L., Mondino, P., and Stadnik, M. 2015. New insight into the characterization of Colletotrichum species associated with apple diseases in southern Brazil and Uruguay. Fungal Biol. 119:229-244.

Wehmeyer, L. E. 1933. The genus Diaporthe Nitschke and its segregates. Univ Michigan Stud., Sci. Ser. 9:1-349.

White, T. J., Bruns, T., Lee, S., and Taylor, J. 1990. Amplification and direct sequencing of fungal ribosomal RNA genes for phylogenetics. Pages 315-322 in: PCR Protocols, A Guide to Methods and Applications. M. A Innis, D. H. Gelfand, J. J. Sninsky, and T. J. White, eds. Academic Press, San Diego, CA

Woudenberg, J. H. C., Seidl, M. F., Groenewald, J. Z., Vries, M., Stielow, J. B., Thomma, B., and Crous, P. W. 2015. Alternaria section Alternaria: species, formae speciales or pathotypes. Stud. Mycol. 82:1-21.

Wu, D., Zhang, D. H., Timko, M. P., Li, M. Y., and Liang, G. L. 2017. First report of Epicoccum nigrum causing brown leaf spot of loquat in Southwestern China Plant Dis. 101:1553.

Yang, Q., Fan, X. L., Du, Z., and Tian, C. M. 2017. Diaporthe juglandicola sp nov. (Diaporthales, Ascomycetes) evidenced by morphological characters and phylogenetic analysis. Mycosphere 8:817-826.

Zhang, C., Wang, W. Z., Diao, Y. Z., and Liu, X. L. 2016. First report of Diaporthe nobilis causing fruit decay of pepper in China. Plant Dis. 100:1948.

Zhu, X., and Xiao, C. L. 2015. Phylogenetic, morphological, and pathogenic characterization of Alternaria species associated with fruit rot of blueberry in California. Phytopathology 105:1555-1567. 